https://helda.helsinki.fi

\title{
Gaussian mixture vector autoregression
}

\section{Kalliovirta, Leena Kaarina}

2016-06

Kalliovirta , L K , Meitz , M H \& Saikkonen , P J 2016 , ' Gaussian mixture vector

autoregression ' , Journal of Econometrics , vol. 192 , no. 2 , pp. 485-498 . https://doi.org/10.1016/j.jeconom.2016.02

http://hdl.handle.net/10138/236799

https://doi.org/10.1016/j.jeconom.2016.02.012

cc_by_nc_nd

acceptedVersion

Downloaded from Helda, University of Helsinki institutional repository.

This is an electronic reprint of the original article.

This reprint may differ from the original in pagination and typographic detail.

Please cite the original version. 


\title{
Gaussian Mixture Vector Autoregression*
}

\author{
Leena Kalliovirta \\ University of Helsinki \\ Natural Resources Institute Finland
}

\author{
Mika Meitz \\ University of Helsinki
}

\author{
Pentti Saikkonen \\ University of Helsinki
}

January 5, 2016

\begin{abstract}
This paper proposes a new nonlinear vector autoregressive (VAR) model referred to as the Gaussian mixture vector autoregressive (GMVAR) model. The GMVAR model belongs to the family of mixture vector autoregressive models and is designed for analyzing time series that exhibit regime-switching behavior. The main difference between the GMVAR model and previous mixture VAR models lies in the definition of the mixing weights that govern the regime probabilities. In the GMVAR model the mixing weights depend on past values of the series in a specific way that has very advantageous properties from both theoretical and practical point of view. A practical advantage is that there is a wide diversity of ways in which a researcher can associate different regimes with specific economically meaningful characteristics of the phenomenon modeled. A theoretical advantage is that stationarity and ergodicity of the underlying stochastic process are straightforward to establish and, contrary to most other nonlinear autoregressive models, explicit expressions of low order stationary marginal distributions are known. These theoretical properties are used to develop an asymptotic theory of maximum likelihood estimation for the GMVAR model whose practical usefulness is illustrated in a bivariate setting by examining the relationship between the EUR-USD exchange rate and a related interest rate data.

Keywords: mixture models, nonlinear vector autoregressive models, regime switching

JEL Classification: C32
\end{abstract}

*The authors thank the Academy of Finland (LK, MM, and PS), the OP-Pohjola Group Research Foundation (LK, MM, and PS), and Finnish Cultural Foundation (PS) for financial support. The paper has benefited from useful comments and suggestions made by the co-editor and three anonymous referees. Contact addresses: Leena Kalliovirta, Department of Political and Economic Studies, University of Helsinki, P. O. Box 17, FI-00014 University of Helsinki, Finland, or Natural Resources Institute Finland (Luke), Viikinkaari 4, FI-00790 Helsinki, Finland; e-mail: leena.kalliovirta@luke.fi. Mika Meitz, Department of Political and Economic Studies, University of Helsinki, P. O. Box 17, FI-00014 University of Helsinki, Finland; e-mail: mika.meitz@helsinki.fi. Pentti Saikkonen, Department of Mathematics and Statistics, University of Helsinki, P. O. Box 68, FI-00014 University of Helsinki, Finland; e-mail: pentti.saikkonen@helsinki.fi. 


\section{Introduction}

The vector autoregressive (VAR) model is one of the main tools used to analyze economic time series. Quite often, the VAR model is assumed linear, although both economic theory and previous empirical evidence may suggest that a nonlinear VAR model could be more appropriate. One popular nonlinear VAR model is the Markov switching VAR (MS-VAR) model that is designed to describe time series that switch between two or more regimes with each regime having the dynamics of a linear VAR model. In most applications, the regime switches are determined by a latent indicator variable that follows a timehomogeneous Markov chain with the transition probabilities depending on the most recent regime but not on past observations (see, e.g., Krolzig (1997) and Sims, Waggoner, and Zha (2008)). More general time-inhomogeneous MS-VAR models, where the transition probabilities depend both on the most recent regime and on past observations, have also been considered (see, e.g., Ang and Bekaert (2002)).

In this paper, we are interested in mixture VAR (MVAR) models. These models can be viewed as special cases of general time-inhomogeneous MS-VAR models from which they are obtained with suitable parameter restrictions. They differ from the commonly used time-homogeneous MS-VAR models in that the transition probabilities do not depend on the most recent regime, but instead on past observations. An equivalent formulation of MVAR models (explaining the nomenclature 'mixture') is to specify the conditional distribution of the process as a mixture of (typically) Gaussian conditional distributions of linear VAR models. Different models are obtained by different specifications of the mixing weights. Univariate mixture autoregressive models were introduced by Le, Martin, and Raftery (1996) and further developed by Wong and Li (2000, 2001a,b) (for further references, see Kalliovirta, Meitz, and Saikkonen (2015); for mixture autoregressions in Bayesian framework, see, e.g., Villani, Kohn, and Giordani (2009)). Extensions to the vector case with economic applications involving inflation, interest rates, stock prices, and exchange rates have been presented by Lanne (2006), Fong, Li, Yau, and Wong (2007), Bec, Rahbek, and Shephard (2008), and Dueker, Psaradakis, Sola, and Spagnolo (2011).

In this paper, we propose a new mixture VAR model referred to as the Gaussian mixture vector autoregressive (GMVAR) model. This model is a multivariate generalization of a similar univariate model introduced in Kalliovirta et al. (2015). The specific formulation of the GMVAR model turns out to have very convenient theoretical implications. To highlight this point, first recall a property that makes the stationary linear Gaussian VAR model different from most, if not nearly all, of its nonlinear alternatives, namely that 
the probability structure of the underlying stochastic process is fully known and can be described by Gaussian densities. In nonlinear VAR (and also nonlinear AR) models the situation is typically very different: the conditional distribution is known by construction but what is usually known beyond that is only the existence of a stationary distribution and finiteness of some of its moments. In the GMVAR model, stationarity of the underlying stochastic process is a simple consequence of the definition of the model. Moreover, letting $p$ denote the autoregressive order of the model (see Section 2), the stationary distribution of $p+1$ consecutive (vector valued) observations is known to be a mixture of multivariate Gaussian distributions with constant mixing weights and known structure for the mean and covariance matrix of the component distributions, whereas the conditional distribution is a multivariate Gaussian mixture with time varying mixing weights. Thus, similarly to the linear Gaussian VAR model, and contrary to (at least most) other nonlinear VAR models, the structure of stationary marginal distributions consisting of $p+1$ observations or less is fully known in the GMVAR model.

In order to interpret a multivariate regime-switching model one typically aims at associating different economically meaningful regimes with different states of economic variables, such as high or low level of inflation, interest rate, or asset return. An appealing feature of the GMVAR model is that, due to the specific structure of the mixing weights, the researcher can associate different regimes with different characteristics of the phenomenon modeled. Moreover, in the GMVAR model switches between regimes are allowed to depend not only on, say, the level of past observations, but on their entire distribution. Thus, in addition to regime switches taking place in periods of high/low levels of the considered series, the GMVAR model can also allow for regime switches taking place in periods of high/low variability, or high/low temporal dependence, and combinations of all these. These convenient features are illustrated in our empirical example, which also demonstrates promising forecasting power of the GMVAR model.

We believe that introducing the GMVAR model makes a useful addition to the literature on multivariate regime-switching models. This is mainly due to the formulation of the model which, in addition to the attractive properties already discussed, has the following implications. First, the regime-switching mechanism is parsimonious and its form becomes automatically specified once the number of regimes and the order of the model are chosen; there is no need to find out which lagged values of the considered series are used to model the regime-switching mechanism and in what form they should be included in the model. Second, conditions that guarantee stationarity (and ergodicity) of the model are entirely similar to those in linear VAR models and they are also necessary 
in the sense of not overly restricting the parameter space of the model. These conditions are therefore both sharp and easy to check, and there is no need to use simulation to find out whether an estimated model fulfills the stationarity condition.

The plan of the paper is as follows. Section 2 discusses general mixture VAR models. Section 3 introduces the new GMVAR model, discusses its theoretical properties, and establishes the consistency and asymptotic normality of the maximum likelihood estimator. Section 4 presents an empirical example with exchange rate and interest rate data, discusses issues of model building, and compares the forecasting performance of the GMVAR model to other linear and nonlinear VAR models. Section 5 concludes, and an Appendix contains some technical derivations. A 'Supplementary Appendix' (available from the authors) contains additional material omitted from the paper.

Finally, a word on notation. We use vec $(A)$ to denote a column vector obtained by stacking the columns of the matrix $A$ one below another. If $A$ is a symmetric matrix then $\operatorname{vech}(A)$ is a column vector obtained by stacking the columns of $A$ from the principal diagonal downwards (including elements on the diagonal). The usual notation $A \otimes B$ is used for the Kronecker product of the matrices $A$ and $B$. To simplify notation, we shall write $z=\left(z_{1}, \ldots, z_{m}\right)$ for the (column) vector $z$ where the components $z_{i}$ may be either scalars or vectors (or both). For any scalar, vector, or matrix $x$, the Euclidean norm is denoted by $|x|$.

\section{Multivariate mixture autoregressive models}

Let $y_{t}(t=1,2, \ldots)$ be the $d$-dimensional time series of interest, and let $\mathcal{F}_{t-1}=\sigma\left(y_{s}, s<t\right)$ denote the $\sigma$-algebra generated by past $y_{t}$ 's. We use $P_{t-1}(\cdot)$ to signify the conditional probability of the indicated event given $\mathcal{F}_{t-1}$. In a general multivariate mixture autoregressive model with $M$ mixture components (or regimes) the $y_{t}$ 's are assumed to be generated by

$$
y_{t}=\sum_{m=1}^{M} s_{t, m}\left(\mu_{m, t}+\Omega_{m}^{1 / 2} \varepsilon_{t}\right),
$$

where the following conditions hold.

\section{Condition 1.}

(a) For each $m=1, \ldots, M, \mu_{m, t}=\phi_{m, 0}+\sum_{i=1}^{p} A_{m, i} y_{t-i}$ and $\Omega_{m}$ is positive definite.

(b) $\varepsilon_{t}(d \times 1)$ is a sequence of independent standard multivariate normal random vectors $\left(\varepsilon_{t} \sim N I D\left(0, I_{d}\right)\right)$ such that $\varepsilon_{t}$ is independent of $\left\{y_{s}, s<t\right\}$. 
(c) $\boldsymbol{s}_{t}=\left(s_{t, 1}, \ldots, s_{t, M}\right)$ is a sequence of (unobserved) random vectors such that for each $t$, exactly one of its components takes the value one and others equal zero, with $\left(\mathcal{F}_{t-1}\right.$-measurable) conditional probabilities $P_{t-1}\left(s_{t, m}=1\right)=\alpha_{m, t}, m=1, \ldots, M$, that satisfy $\sum_{m=1}^{M} \alpha_{m, t}=1$.

(d) Conditional on $\mathcal{F}_{t-1}, \boldsymbol{s}_{t}$ and $\varepsilon_{t}$ are independent.

For later developments, we collect the unknown parameters introduced above in the vectors $\boldsymbol{\vartheta}_{m}=\left(\phi_{m, 0}, \boldsymbol{\phi}_{m}, \sigma_{m}\right)$ with $\boldsymbol{\phi}_{m}=\left(\operatorname{vec}\left(A_{m, 1}\right), \ldots, \operatorname{vec}\left(A_{m, p}\right)\right)$ and $\sigma_{m}=\operatorname{vech}\left(\Omega_{m}\right)$ $(m=1, \ldots, M)$.

The conditional probabilities $\alpha_{m, t}$ in Condition 1(c) are referred to as mixing weights (this nomenclature will be made clear shortly). They can be thought of as probabilities that determine which one of the $M$ VAR components in (1) generates the next observation. To complete the definition of an MVAR model, the mixing weights need to be specified. Our specification as well as some alternatives are discussed in Section 3.1.

Using equation (1) and Condition 1 the conditional density function of $y_{t}$ given its past, $f\left(\cdot \mid \mathcal{F}_{t-1}\right)$, is obtained as

$$
f\left(y_{t} \mid \mathcal{F}_{t-1}\right)=\sum_{m=1}^{M} \alpha_{m, t}(2 \pi)^{-d / 2} \operatorname{det}\left(\Omega_{m}\right)^{-1 / 2} \exp \left\{-\frac{1}{2}\left(y_{t}-\mu_{m, t}\right)^{\prime} \Omega_{m}^{-1}\left(y_{t}-\mu_{m, t}\right)\right\} .
$$

Thus, the distribution of $y_{t}$ given its past is specified as a mixture of multivariate normal densities with time varying mixing weights $\alpha_{m, t}$. The conditional mean and covariance matrix of $y_{t}$ given $\mathcal{F}_{t-1}$ can be expressed as

$$
E\left[y_{t} \mid \mathcal{F}_{t-1}\right]=\sum_{m=1}^{M} \alpha_{m, t} \mu_{m, t}=\sum_{m=1}^{M} \alpha_{m, t}\left(\phi_{m, 0}+\sum_{i=1}^{p} A_{m, i} y_{t-i}\right)
$$

and

$$
\operatorname{Cov}\left[y_{t} \mid \mathcal{F}_{t-1}\right]=\sum_{m=1}^{M} \alpha_{m, t} \Omega_{m}+\sum_{m=1}^{M} \alpha_{m, t}\left(\mu_{m, t}-\sum_{n=1}^{M} \alpha_{n, t} \mu_{n, t}\right)\left(\mu_{m, t}-\sum_{n=1}^{M} \alpha_{n, t} \mu_{n, t}\right)^{\prime}
$$

These expressions are valid for any specification of the mixing weights $\alpha_{m, t}$.

\section{Gaussian Mixture Vector Autoregressive (GMVAR) model}

\subsection{Definition}

The GMVAR model is based on a particular choice of the mixing weights $\alpha_{m, t}$ in Condition 1(c). This choice is similar to that used by Glasbey (2001) and Kalliovirta et al. (2015) in 
a univariate setting. In order to define these mixing weights we first use the parameters $\phi_{m, 0}, A_{m, i}$, and $\Omega_{m}$ (see (1) and Condition 1(a)) to define the $M$ auxiliary linear Gaussian VAR processes

$$
\nu_{m, t}=\phi_{m, 0}+\sum_{i=1}^{p} A_{m, i} \nu_{m, t-i}+\Omega_{m}^{1 / 2} \varepsilon_{t}, \quad m=1, \ldots, M,
$$

where the coefficient matrices $A_{m, i}$ are assumed to satisfy

$$
\operatorname{det} A_{m}(z)=\operatorname{det}\left(I_{d}-\sum_{i=1}^{p} A_{m, i} z^{i}\right) \neq 0 \quad \text { for }|z| \leq 1, \quad m=1, \ldots, M \text {. }
$$

This condition implies that the processes $\nu_{m, t}(d \times 1)$ are stationary and that each component model in (1) satisfies the usual stationarity condition of a linear VAR model. We also need the density function of the (Gaussian) random vector $\boldsymbol{\nu}_{m, t}=\left(\nu_{m, t}, \ldots, \nu_{m, t-p+1}\right)$ $(d p \times 1)(m=1, \ldots, M)$ given by

$\mathrm{n}_{d p}\left(\boldsymbol{\nu}_{m, t} ; \boldsymbol{\vartheta}_{m}\right)=(2 \pi)^{-d p / 2} \operatorname{det}\left(\boldsymbol{\Sigma}_{m, p}\right)^{-1 / 2} \exp \left\{-\frac{1}{2}\left(\boldsymbol{\nu}_{m, t}-\mathbf{1}_{p} \otimes \mu_{m}\right)^{\prime} \boldsymbol{\Sigma}_{m, p}^{-1}\left(\boldsymbol{\nu}_{m, t}-\mathbf{1}_{p} \otimes \mu_{m}\right)\right\}$,

where $\mathbf{1}_{p}=(1, \ldots, 1)(p \times 1), \mu_{m}=A_{m}^{-1}(1) \phi_{m, 0}$, and the covariance matrix $\boldsymbol{\Sigma}_{m, p}$ is a function of $A_{m, i}, i=1, \ldots, p$, and $\Omega_{m}$, and hence of the parameters $\phi_{m}$ and $\sigma_{m}$ (for details, see Lütkepohl (2005, eqn. (2.1.39))).

Now we can specify our choice for the mixing weights $\alpha_{m, t}$. Using the vector $\boldsymbol{y}_{t-1}=$ $\left(y_{t-1}, \ldots, y_{t-p}\right)(d p \times 1)$ and the multivariate Gaussian densities in (6), we set

$$
\alpha_{m, t}=\frac{\alpha_{m} \mathbf{n}_{d p}\left(\boldsymbol{y}_{t-1} ; \boldsymbol{\vartheta}_{m}\right)}{\sum_{n=1}^{M} \alpha_{n} \mathbf{n}_{d p}\left(\boldsymbol{y}_{t-1} ; \boldsymbol{\vartheta}_{n}\right)},
$$

where the $\alpha_{m} \in(0,1), m=1, \ldots, M$, are unknown parameters satisfying $\sum_{m=1}^{M} \alpha_{m}=1$. (Clearly, the coefficients $\alpha_{m, t}$ are measurable functions of $\boldsymbol{y}_{t-1}=\left(y_{t-1}, \ldots, y_{t-p}\right)$ and satisfy $\sum_{m=1}^{M} \alpha_{m, t}=1$ for all $t$.) Equation (1), Condition 1, and (7) define the Gaussian Mixture Vector Autoregressive model or the GMVAR model. We use the abbreviation $\operatorname{GMVAR}(p, M)$ when the autoregressive order and number of component models need to be emphasized. The unknown parameters to be estimated are collected in the vector $\boldsymbol{\theta}=\left(\boldsymbol{\vartheta}_{1}, \ldots, \boldsymbol{\vartheta}_{M}, \alpha_{1}, \ldots, \alpha_{M-1}\right)\left(\left(M\left(d^{2} p+d+d(d+1) / 2+1\right)-1\right) \times 1\right)$; the coefficient $\alpha_{M}$ is not included due to the restriction $\sum_{m=1}^{M} \alpha_{m}=1$.

As already mentioned, the specification of the mixing weights in (7) is analogous to that used by Glasbey (2001) and Kalliovirta et al. (2015) in a univariate setting; indeed, when $d=1$ the GMVAR model reduces to the GMAR model of Kalliovirta et al. (2015). Of 
previous multivariate mixture autoregressive models, Fong et al. (2007) consider constant mixing weights, that is, $\alpha_{m, t}=\alpha_{m}$, whereas Bec et al. (2008) study a two component model $(M=2)$ with time-varying mixing weights specified via a logistic function (cf. the univariate model of Wong and $\mathrm{Li}(2001 \mathrm{~b})$ ). The time-varying mixing weights used by Dueker et al. (2011) in their C-MSTAR model are based on a formula that is formally similar to (7) except that instead of density functions these authors employ cumulative distribution functions of the multinormal distribution. ${ }^{1}$ A particular feature of their model is that the number of regimes, $M$, is always determined by the dimension of the model, $d$, so that $M=d^{2}$. Even when the dimension $d$ is fairly small, say three or four, this makes the number of regimes, and hence the number of parameters to be estimated quite large, and special measures may be called for to facilitate estimation in practice (see Dueker et al. (2011, Footnote 5)). This is in contrast to our GMVAR model where the number of regimes is not related to the dimension of the model and the mixing mechanism is modeled in a parsimonious fashion in that only the parameters $\alpha_{1}, \ldots, \alpha_{M-1}$ are involved.

Even though parsimony is an important feature of our mixing weights, its major advantage is theoretical attractiveness, as discussed in the next subsection.

\subsection{Theoretical properties}

Given the specification of the mixing weights $\alpha_{m, t}$ in (7), the conditional distribution of $\boldsymbol{y}_{t}$ given $\mathcal{F}_{t-1}$ only depends on $\boldsymbol{y}_{t-1}$, implying that $\boldsymbol{y}_{t}$ is Markovian. This fact is formally stated in the following theorem which shows that there exists a choice of initial values $\boldsymbol{y}_{0}$ such that $\boldsymbol{y}_{t}$ is a stationary and ergodic Markov chain. An explicit expression for the stationary distribution is also provided. The following theorem is proved in the Appendix.

Theorem 1. Consider the GMVAR process $y_{t}$ generated by (1) and (7), and assume that Condition 1 and condition (5) are satisfied. Then $\boldsymbol{y}_{t}=\left(y_{t}, \ldots, y_{t-p+1}\right)$ is a Markov chain on $\mathbb{R}^{d p}$ with a stationary distribution characterized by the density

$$
f(\boldsymbol{y} ; \boldsymbol{\theta})=\sum_{m=1}^{M} \alpha_{m} \mathrm{n}_{d p}\left(\boldsymbol{y} ; \boldsymbol{\vartheta}_{m}\right) .
$$

Moreover, $\boldsymbol{y}_{t}$ is ergodic.

\footnotetext{
${ }^{1}$ According to the authors their model belongs to the family of multivariate STAR models and this interpretation is indeed consistent with the initial definition of the model (see equations (3) and (4) in Dueker et al. (2011)). However, we treat the model as a mixture model because the likelihood function used to fit the model to data is determined by (not necessarily Gaussian) conditional density functions that are of the mixture form (2) (see Section 4.1 in Dueker et al. (2011)).
} 
Theorem 1 is an analog of the corresponding result obtained by Kalliovirta et al. (2015) in the univariate case $d=1$. It shows that the stationary distribution of $\boldsymbol{y}_{t}$ is a mixture of $M$ multinormal distributions with constant mixing weights $\alpha_{m}$ that appear in the time varying mixing weights $\alpha_{m, t}$ defined in (7). Consequently, all moments of the stationary distribution exist and are finite. In fact, as can be seen from the proof of Theorem 1, the stationary distribution of the $d(p+1)$-dimensional random vector $\left(y_{t}, \boldsymbol{y}_{t-1}\right)$ is also a Gaussian mixture with density of the same form as in (8) or, specifically, $\sum_{m=1}^{M} \alpha_{m} \mathrm{n}_{d(p+1)}\left((y, \boldsymbol{y}) ; \boldsymbol{\vartheta}_{m}\right)$. This implies that the marginal distributions of this Gaussian mixture belong to the same family ${ }^{2}$ and also that explicit expressions for the mean, variance, and first $p$ autocovariances of the stationary version of the process $y_{t}$ can easily be derived (cf. Kalliovirta et al. (2015, p. 251)).

As discussed by Kalliovirta et al. (2015), it is quite exceptional that complete knowledge of the stationary distribution of a nonlinear autoregressive model is available and that readily verifiable conditions that define the parameter space can be used to obtain a relatively simple proof for stationarity and ergodicity. Similarly to their univariate counterparts, Theorem 1 and the definition of the model suggest that the GMVAR model can be quite flexible in describing various nonlinear and non-Gaussian features encountered in time series data (for details, see Section 2 of Kalliovirta et al. (2015)).

\subsection{Interpretation of the mixing weights $\alpha_{m}$ and $\alpha_{m, t}$}

Unless otherwise stated, we assume the stationary version of the GMVAR process in this and the next subsection. According to Theorem 1, the parameter $\alpha_{m}(m=1, \ldots, M)$ has an immediate interpretation as the unconditional probability of the random vector $\boldsymbol{y}_{t}=\left(y_{t}, \ldots, y_{t-p+1}\right)$ being generated from a distribution with density $\mathrm{n}_{d p}\left(\boldsymbol{y} ; \boldsymbol{\vartheta}_{m}\right)$, that is, from the $m$ th component of the Gaussian mixture characterized in (8). Consequently, $\alpha_{m}(m=1, \ldots, M)$ also represents the unconditional probability of the component $y_{t}$ being generated from a distribution with density $\mathrm{n}_{d}\left(y ; \boldsymbol{\vartheta}_{m}\right)$, the $m$ th component of the $(d-$ dimensional) Gaussian mixture density $\sum_{m=1}^{M} \alpha_{m} \mathbf{n}_{d}\left(y ; \boldsymbol{\vartheta}_{m}\right)$ where $\mathbf{n}_{d}\left(y ; \boldsymbol{\vartheta}_{m}\right)$ is the density function of a Gaussian random vector with mean $\mathbf{1}_{p} \otimes \mu_{m}$ and covariance matrix $\boldsymbol{\Gamma}_{m, 0}$.

It can also be shown that $\alpha_{m}$ represents the unconditional probability of (the $d-$ dimensional) $y_{t}$ being generated from the $m$ th VAR component in (1) whereas $\alpha_{m, t}$ represents the corresponding conditional probability $P_{t-1}\left(s_{t, m}=1\right)=\alpha_{m, t}$. This conditional

\footnotetext{
${ }^{2}$ Note, however, that this does not hold in higher dimensions so that the stationary distribution of $\left(y_{t+1}, y_{t}, \boldsymbol{y}_{t-1}\right)$, for example, is not a Gaussian mixture.
} 
probability depends on the (relative) size of the product $\alpha_{m} \mathrm{n}_{d p}\left(\boldsymbol{y}_{t-1} ; \boldsymbol{\vartheta}_{m}\right)$, the numerator of the expression defining $\alpha_{m, t}$ (see (7)). The latter factor of this product, $\mathrm{n}_{d p}\left(\boldsymbol{y}_{t-1} ; \boldsymbol{\vartheta}_{m}\right)$, can be interpreted as the likelihood of the $m$ th autoregressive component in (1) based on the observation $\boldsymbol{y}_{t-1}$. Thus, the larger this likelihood is the more likely it is to observe $y_{t}$ from the $m$ th autoregressive component. However, the product $\alpha_{m} \mathrm{n}_{d p}\left(\boldsymbol{y}_{t-1} ; \boldsymbol{\vartheta}_{m}\right)$ is also affected by the former factor $\alpha_{m}$ or the weight of $\mathrm{n}_{d p}\left(\boldsymbol{y}_{t-1} ; \boldsymbol{\vartheta}_{m}\right)$ in the stationary mixture distribution of $\boldsymbol{y}_{t-1}$ (evaluated at $\boldsymbol{y}_{t-1}$; see (8)). Specifically, even though the likelihood of the $m$ th autoregressive component in (1) is, for example, large, a small value of $\alpha_{m}$ attenuates its effect so that the likelihood of observing $y_{t}$ from the $m$ th autoregressive component can be small. This seems intuitively natural because, for example, a small weight of $\mathrm{n}_{d p}\left(\boldsymbol{y}_{t-1} ; \boldsymbol{\vartheta}_{m}\right)$ in the stationary mixture distribution of $\boldsymbol{y}_{t-1}$ means that observations cannot be generated by the $m$ th autoregressive component too frequently.

The preceding discussion highlights the fact that the GMVAR model associates the mixing weights or the regime probabilities $P_{t-1}\left(s_{t, m}=1\right)=\alpha_{m, t}$ with observable economic characteristics through the density functions $\mathrm{n}_{d p}\left(\boldsymbol{y}_{t-1} ; \boldsymbol{\vartheta}_{m}\right)$. In particular, and in contrast to existing mixture VAR models, the specific form of the mixing weights in the GMVAR model therefore allows the regime probabilities $\alpha_{m, t}$ to depend on the entire distribution of $p$ past observations and not only on some of their specific features such as levels.

\subsection{Parameter estimation}

The parameters of the GMVAR model can be estimated by the method of maximum likelihood (ML). As the stationary distribution of the GMVAR process is known it is even possible to make use of initial values and construct the exact likelihood function and obtain exact ML estimates. Assuming the observed data $y_{-p+1}, \ldots, y_{0}, y_{1}, \ldots, y_{T}$ and stationary initial values the log-likelihood function takes the form

$$
\mathrm{L}_{T}(\boldsymbol{\theta})=\log \left(\sum_{m=1}^{M} \alpha_{m} \mathrm{n}_{d p}\left(\boldsymbol{y}_{0} ; \boldsymbol{\vartheta}_{m}\right)\right)+\sum_{t=1}^{T} l_{t}(\boldsymbol{\theta})
$$

where

$$
\begin{aligned}
l_{t}(\boldsymbol{\theta})=\log \left(\sum_{m=1}^{M} \alpha_{m, t}\right. & (\boldsymbol{\theta})(2 \pi)^{-d / 2} \operatorname{det}\left(\Omega_{m}\right)^{-1 / 2} \\
\times & \left.\exp \left\{-\frac{1}{2}\left(y_{t}-\mu_{m, t}\left(\boldsymbol{\vartheta}_{m}\right)\right)^{\prime} \Omega_{m}^{-1}\left(y_{t}-\mu_{m, t}\left(\boldsymbol{\vartheta}_{m}\right)\right)\right\}\right) .
\end{aligned}
$$

Here dependence of the mixing weights $\alpha_{m, t}$ and the conditional expectations $\mu_{m, t}$ of the component models on the parameters is made explicit (see (7) and Condition 1(a)). In the 
expression (9) it has been assumed that the initial values in the vector $\boldsymbol{y}_{0}$ are generated by the stationary distribution. If this assumption seems inappropriate one can condition on initial values and drop the first term on the right hand side of (9). In what follows we assume estimation is performed based on this conditional likelihood, namely

$$
L_{T}(\boldsymbol{\theta})=T^{-1} \sum_{t=1}^{T} l_{t}(\boldsymbol{\theta}),
$$

which we, for convenience, have also scaled with the sample size. Maximizing the conditional log-likelihood function $L_{T}(\boldsymbol{\theta})$ with respect to the parameter vector $\boldsymbol{\theta}$ yields the ML estimate denoted by $\hat{\boldsymbol{\theta}}_{T}$ (a similar notation is used for components of $\hat{\boldsymbol{\theta}}_{T}$ ).

Investigation of the asymptotic properties of the ML estimator $\hat{\boldsymbol{\theta}}_{T}$ requires further assumptions. The unknown model parameters are collected in the vector $\boldsymbol{\theta}=(\boldsymbol{\vartheta}, \boldsymbol{\alpha})=$ $\left(\boldsymbol{\vartheta}_{1}, \ldots, \boldsymbol{\vartheta}_{M}, \alpha_{1}, \ldots, \alpha_{M-1}\right)$ taking values in the parameter space $\Theta$. The parameter space $\Theta$ needs to be constrained in various ways. Earlier we already mentioned the stationarity conditions (5) and the positive definiteness of the covariance matrices $\Omega_{m}(m=1, \ldots, M)$ that are assumed to hold. Throughout we assume that the number of mixture components is known, and this also entails the requirement that the coefficients $\alpha_{m}, m=1, \ldots, M$, used to define the mixing weights are all strictly positive (and strictly less than unity). Further restrictions are required to ensure identification of the model parameters. In the proof of Theorem 2 below, the identification of GMVAR models is established by using the results of Yakowitz and Spragins (1968) on the identification of finite mixtures of (in our case) multivariate Gaussian distributions. Essentially, what is required is that the $M$ component models cannot be 'relabeled' and the same GMVAR model obtained. A sufficient condition ensuring this is

$$
\alpha_{1}>\cdots>\alpha_{M}>0 \text { and } \boldsymbol{\vartheta}_{i}=\boldsymbol{\vartheta}_{j} \text { only if } 1 \leq i=j \leq M \text {. }
$$

We summarize the restrictions imposed on the parameter space as follows.

Assumption 1. The true parameter value $\boldsymbol{\theta}_{0}$ is an interior point of $\Theta$, where $\Theta$ is a compact subset of $\left\{\boldsymbol{\theta} \in \mathbb{R}^{M\left(d+p d^{2}+d(d+1) / 2\right)} \times(0,1)^{M-1}:\right.$ (5) and (11) hold, and $\Omega_{m}$ $(m=1, \ldots, M)$ are positive definite $\}$.

The following theorem establishes the strong consistency of the ML estimator.

Theorem 2. Suppose $y_{t}$ are generated by the stationary and ergodic GMVAR process of Theorem 1 and that Assumption 1 holds. Then the maximum likelihood estimator $\hat{\boldsymbol{\theta}}_{T}$ is strongly consistent, that is, $\hat{\boldsymbol{\theta}}_{T} \rightarrow \boldsymbol{\theta}_{0}$ a.s. 
To simplify the proof Theorem 2 assumes stationary initial values (relaxing this assumption is possible at the cost of a longer and more complicated proof). A similar remark applies to our next theorem below where we show that the ML estimator $\hat{\boldsymbol{\theta}}_{T}$ is asymptotically normally distributed. To this end, Lemmas 1-3 in the Appendix establish some of the major ingredients required for proving this result, namely (i) that the score vector (evaluated at the true parameter value) is a (square integrable) martingale difference sequence and thus obeys a central limit theorem, (ii) that the Hessian matrix of the log-likelihood function converges uniformly in some neighborhood of the true parameter value, and (iii) that, when evaluated at the true parameter value, the limiting covariance matrix of the score vector equals the negative of the expected Hessian. The last main ingredient required, positive definiteness of the information matrix, is contained in the following assumption.

Assumption 2. The matrix $\mathcal{I}\left(\boldsymbol{\theta}_{0}\right)=E\left[\frac{\partial l_{t}\left(\boldsymbol{\theta}_{0}\right)}{\partial \boldsymbol{\theta}} \frac{\partial l_{t}\left(\boldsymbol{\theta}_{0}\right)}{\partial \boldsymbol{\theta}^{\prime}}\right]$ is positive definite.

Verification of Assumption 2 in the GMVAR model considered is challenging, one reason for this being the rather complex expressions the partial derivatives of the mixing weights $\alpha_{m, t}$ have. A similar assumption is made by Dueker et al. (2011, condition C.9 on p. 324), who show that, under appropriate 'high level' regularity conditions, the usual results of consistency and asymptotic normality of the ML estimator hold in their mixture model. The following theorem establishes asymptotic normality of the ML estimator in our GMVAR model.

Theorem 3. Suppose $y_{t}$ are generated by the stationary and ergodic GMVAR process of Theorem 1 and that Assumptions 1 and 2 hold. Then

$$
T^{1 / 2}\left(\hat{\boldsymbol{\theta}}_{T}-\boldsymbol{\theta}_{0}\right) \stackrel{d}{\rightarrow} N\left(0,-\mathcal{J}\left(\boldsymbol{\theta}_{0}\right)^{-1}\right)
$$

where $\mathcal{J}\left(\boldsymbol{\theta}_{0}\right)=E\left[\partial^{2} l_{t}\left(\boldsymbol{\theta}_{0}\right) / \partial \boldsymbol{\theta} \partial \boldsymbol{\theta}^{\prime}\right]=-\mathcal{I}\left(\boldsymbol{\theta}_{0}\right)$ is finite.

Theorem 3 shows that the conventional limiting distribution applies to the ML estimator $\hat{\boldsymbol{\theta}}_{T}$ which (in conjunction with the derivations used in the proof of Theorem 3) implies the applicability of standard likelihood-based tests. It is worth noting, however, that here a correct specification of the number of autoregressive components $M$ is required. In particular, if the number of component models is chosen too large then some parameters of the model are not identified and, consequently, the result of Theorem 3 and the validity of the related tests break down. This particularly happens when one tests for the number 
of component models, an issue discussed in more detail below (see also Dueker, Sola, and Spagnolo (2007), Dueker et al. (2011), and the references therein).

Suppose one is interested in testing the null hypothesis of a linear VAR model (a model where the number of component models $M$ equals 1 ) against a nonlinear GMVAR model (with $M \geq 2$ ) or, more generally, to select the number of component models $M$. In such testing problems, difficulties arise because in a $\operatorname{GMVAR}(p, M)$ model some parameters are not identified if the model reduces to a linear $\operatorname{VAR}(p)$ model. A further complication is that the $\operatorname{GMVAR}(p, M)$ model can be reduced to a $\operatorname{VAR}(p)$ model in several ways. For instance, when $M=2$ a $\operatorname{VAR}(p)$ model is obtained by specifying the null hypothesis either as $\boldsymbol{\vartheta}_{1}=\boldsymbol{\vartheta}_{2}$ or as $\alpha_{1}=1$. In the former case the parameter $\alpha_{1}$ is not identified and in the latter case the parameter $\boldsymbol{\vartheta}_{2}$ is not identified. The latter case also involves the nonstandard feature that under null hypothesis the parameter $\alpha_{1}$ lies on the boundary of the parameter space. These facts indicate that the testing problem is highly nonstandard.

Likelihood-based tests for testing problems similar to those discussed above have recently been studied for mixture or Markov switching type regime switching models, among others, by Cho and White (2007) and Carrasco, Hu, and Ploberger (2014). The former authors develop tests in mixture models where the mixing weights are constant over time, whereas the latter authors discuss tests in Markov switching models in which the transition probabilities depend on past regimes but not on past observations. To our knowledge, tests for the case in which the mixing weights depend on past data have not yet been developed. The complexity of the abovementioned papers suggests, however, that developing such tests may be a major task and is, therefore, left for future research.

Instead of formal tests, in our empirical application we use residual-based diagnostics and information criteria (AIC and BIC) to infer which model fits the data best. Similar approaches have also been used by Fong et al. (2007) and Dueker et al. (2011) in their mixture VAR models. Note, however, that once the number of regimes $M$ in the GMVAR model is (correctly) chosen, standard likelihood-based inference can be used to choose regime-wise autoregressive orders and to test other hypotheses of interest.

\section{Empirical example}

\subsection{Data and preliminary analysis}

We use interest rate and exchange rate data to illustrate how the GMVAR model can describe typical features of financial data and how it improves the in-sample and out-of- 

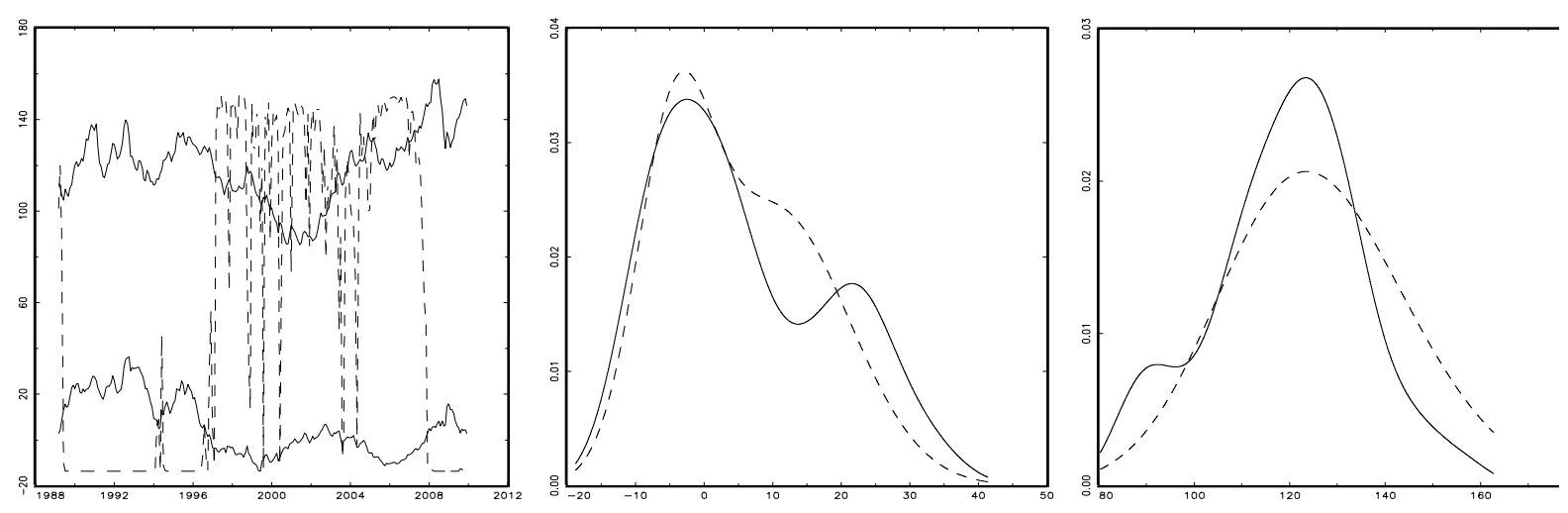

Figure 1: Left: The Euro and U.S. dollar exchange rate series scaled by 100 (upper solid line), the interest rate differential between the Euro area and U.S. scaled by 10 (lower solid line), and scaled mixing weights based on the estimates of the GMVAR model in (12) (dashed line; the scaling is such that $\hat{\alpha}_{1, t}$ equals the maximum (minimum) of the observations when $\hat{\alpha}_{1, t}=1$ $(=0)$ ). Middle: A kernel density estimate of the observations (solid line) and mixture density implied by the same GMVAR model as in the left panel for the interest rate differential series (dashed line). Right: A kernel density estimate of the observations (solid line) and mixture density implied by the same GMVAR model as in the left panel for the exchange rate series (dashed line).

sample fit compared to a linear VAR model and competing regime switching nonlinear VAR models. Our data, retrieved from OECD Statistics, consists of the difference between the monthly Euro area and U.S. long-term government bond yields (referred to as the interest rate differential), $z_{t, 1}$, and the monthly average Euro-U.S. dollar exchange rate, $z_{t, 2}$, from January 1989 to December $2013 .{ }^{3}$ We split the data into an estimation period of 21 years and a forecasting period of 4 years, and analyze slightly transformed data, $\boldsymbol{y}_{t}=\left(10 z_{t, 1}, 100 z_{t, 2}\right)$, to ease the numerical maximization of the likelihood function. Time series plots of the transformed data are shown in Figure 1 for the estimation period that ends on December 2009 (left panel, the solid lines).

Visual inspection of the time series plots of the series (Figure 1, solid lines in the left panel) suggests that the two series exhibit changes at least in levels and potentially also in variability. Kernel estimates of the density functions of the component series (Figure 1, solid lines in the middle and right panels) also suggest the potential presence of multiple

\footnotetext{
${ }^{3}$ The difference between yields of government bonds with 10 years maturity, $z_{t, 1}=i_{t, E U}-i_{t, U S}$, is calculated by the ECB and the Federal Reserve Board; prior to 2001, the Euro area data refer to the "EU11" countries, and afterwards with changing composition eventually to the "EU17" by the end of the data period. The latter series, $z_{t, 2}$, is based on the ECU-USD exchange rate prior to 1999.
} 
regimes and multimodality. These observations are in line with the univariate analyses of the component series in Kalliovirta et al. (2014, 2015). Although the time periods used in these papers differ somewhat from that used here, the obtained results nevertheless lend support to the fact that the component series exhibit regime switching dynamics that could adequately be described by univariate versions of the GMVAR model.

For a multivariate analysis, a natural first step is to check how well conventional linear Gaussian VAR models fit the data. For brevity, we do not present the results of the linear VAR analysis performed (the results are available in the Supplementary Appendix). In summary, BIC suggested a $\operatorname{VAR}(2)$ model which, however, was clearly rejected in that the residuals were found conditionally heteroskedastic, autocorrelated, and non-Gaussian (a similar result was obtained when a VAR(4), suggested by AIC, was tried).

\subsection{The estimated GMVAR model}

When specifying a $\operatorname{GMVAR}(p, M)$ model, it is advisable to begin with low-order models. One reason for this is that if the number of component models $M$ is chosen too large, then some parameters of the model are not identified (see the discussion in Section 3.4 following Theorem 3). For the autoregressive order $p$, the order chosen for the linear VAR model appears a natural initial choice. As Kalliovirta et al. (2014, 2015) found that the component series could adequately be described by univariate versions of the GMVAR model with the autoregressive coefficients restricted the same in each regime, we started our multivariate analysis with a similarly restricted $\operatorname{GMVAR}(2,2)$ model. Thus, we first tried a simple 2-regime GMVAR model with autoregressive order 2 and regimewise intercept terms and error covariance matrices. Estimation based on the conditional likelihood gave the following results:

$$
\begin{aligned}
{\left[\begin{array}{l}
y_{t, 1} \\
y_{t, 2}
\end{array}\right]=} & {\left[\begin{array}{ll}
1.25 & 0.04 \\
(0.07) & (0.04) \\
0.06 & 1.34 \\
(0.10) & (0.06)
\end{array}\right]\left[\begin{array}{l}
y_{t-1,1} \\
y_{t-1,2}
\end{array}\right]+\left[\begin{array}{rr}
-0.29 & -0.05 \\
(0.06) & (0.04) \\
-0.08 & -0.36 \\
(0.10) & (0.06)
\end{array}\right]\left[\begin{array}{l}
y_{t-2,1} \\
y_{t-2,2}
\end{array}\right] } \\
+ & \left(\left[\begin{array}{l}
1.03 \\
(0.51) \\
2.36 \\
(1.34)
\end{array}\right]+\left[\begin{array}{cc}
0.93 & -0.15 \\
(0.15) & (0.27) \\
-0.15 & 5.20 \\
(0.27) & (0.76)
\end{array}\right]^{1 / 2} \hat{\varepsilon}_{t}\right)+s_{t, 2}\left(\left[\begin{array}{l}
1.79 \\
(0.69) \\
3.00 \\
(1.48)
\end{array}\right]+\left[\begin{array}{ll}
5.88 & 3.56 \\
(0.73) & (0.72) \\
3.56 & 9.80 \\
(0.72) & (1.18)
\end{array}\right]^{1 / 2} \hat{\varepsilon}_{t}\right)
\end{aligned}
$$

with the estimate of the mixing weight $\alpha_{1}=P\left(s_{t, 1}=1\right)$ being $\hat{\alpha}_{1}=0.37(0.18)$ and the estimated correlation of the error terms being -0.07 (0.12) in regime 1 and $0.47(0.07)$ in regime 2 (standard errors computed using the Hessian of the log-likelihood function 
are given in parentheses). Based on the maximized values of the likelihood functions and information criteria, this model is clearly preferable to the linear $\operatorname{VAR}(2)$ model. ${ }^{4}$

The time series plot of the estimated time-varying mixing weights $\hat{\alpha}_{1, t}$ is depicted in Figure 1 (dashed line in the left panel). From 1989 until the beginning of 1996, the second regime is clearly dominating. Between 1996 and 2008 the series mostly evolve in the first regime, although there are a few occasions where the probability of the second regime is quite high. After 2008, the series switch back to the second regime. Based on the time series plots of the estimated conditional means and conditional variances of the component series (see Figure A.8 in the Supplementary Appendix; computed according to (3) and (4)), the first regime corresponds to a low mean, low variance regime, whereas the second regime corresponds to a high mean, high variance regime. This is consistent with the time series plots of the series (Figure 1, solid lines in the left panel) as well as with the estimates of the means and variances of the stationary distribution implied by the estimated model (12) (not shown) in that in the first regime these estimates are small compared to their counterparts in the second regime. The time series plot of the estimated conditional correlation between the two series (see Figure A.8 in the Supplementary Appendix; computed according to (4)) has a shape similar to that of an overturned version of the estimated mixing weights $\hat{\alpha}_{1, t}$ (depicted in Figure 1 ). In the first regime the series evolve rather uncorrelated of each other (the estimated conditional correlation during the years 1996-2008 is most of the time between 0 and 0.25 ), whereas in the second regime they are correlated (the estimated conditional correlation before 1996 and after 2008 is near-constant at 0.47).

The estimates of the off-diagonal elements of the autoregressive matrices in (12) are rather small, and for completeness, we also estimated a model in which these elements were restricted to zero. The likelihood ratio test for this restriction had a $p$-value of 0.12 , but as the (quantile) residuals of the restricted model were autocorrelated and the restricted model produced forecasts inferior to those of the unrestricted model, we prefer the unrestricted model. It may be worth noting that even if the off-diagonal elements of the autoregressive matrices were zero the conditional distribution of $y_{t, 1}$, for example, given the past of the two series is not independent of past values of $y_{t, 2}$ (a similar conclusion holds for $\left.y_{t, 2}\right)$. The reason for this is that the mixing weight $\alpha_{1, t}$ depends on $\left(y_{t-1,2}, y_{t-2,2}\right)$. In particular, as the intercept terms and variances in the two regimes differ the conditional expectation $E\left[y_{t, 1} \mid \mathcal{F}_{t-1}\right]$ and the conditional variance $\operatorname{Var}\left[y_{t, 1} \mid \mathcal{F}_{t-1}\right]$ are functions of

\footnotetext{
${ }^{4}$ The maximized value of the log-likelihood function is -1083 , and the AIC and BIC values are 2205 and 2272. The corresponding figures for the linear $\operatorname{VAR}(2)$ model are $-1116,2258$, and 2304.
} 

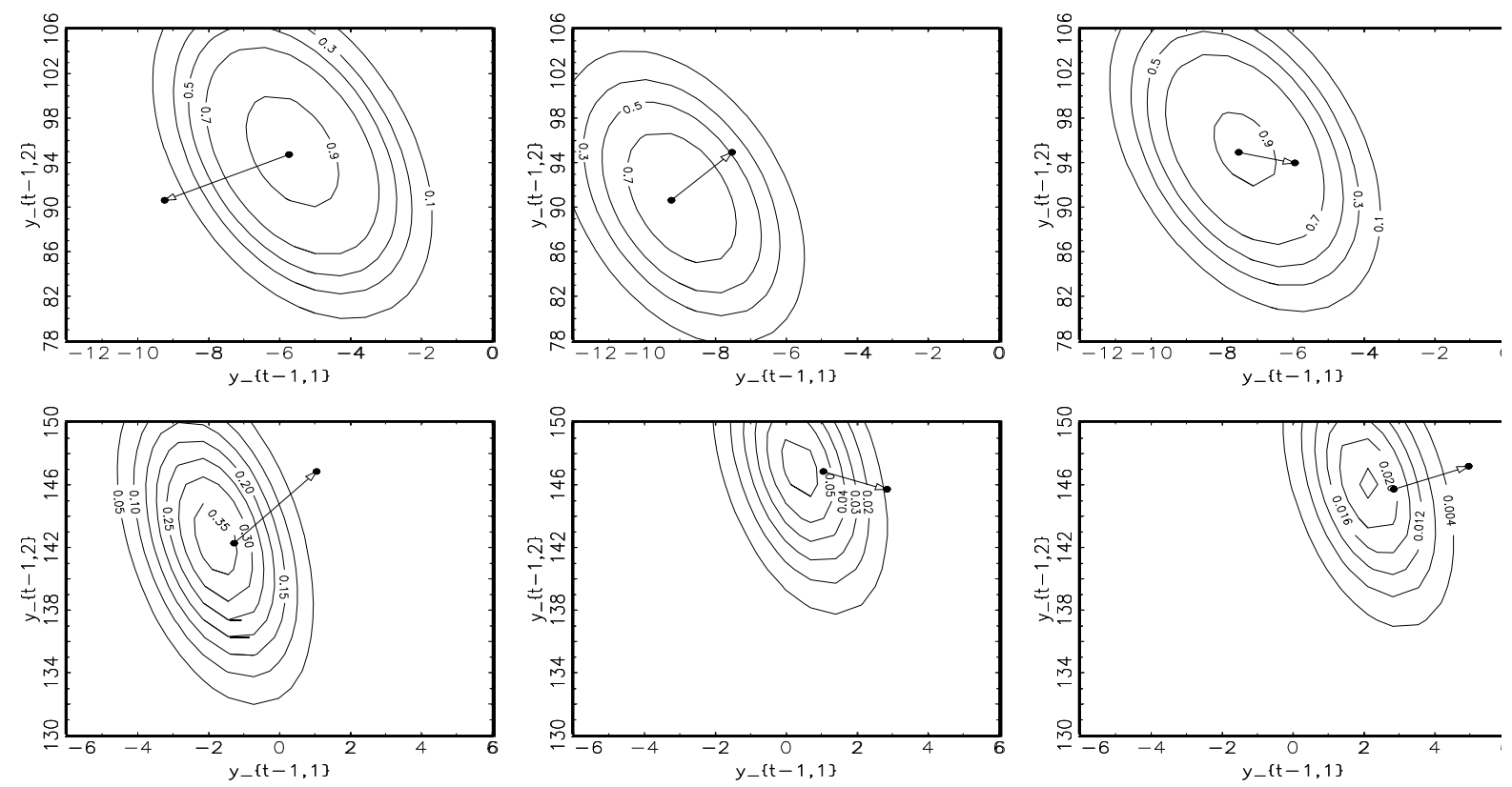

Figure 2: Contour plots of the mixing weights $\hat{\alpha}_{1, t}$ as a function of $y_{t-1}=\left(y_{t-1,1}, y_{t-1,2}\right)$ with $y_{t-2}=\left(y_{t-2,1}, y_{t-2,2}\right)$ fixed and with $y_{t-1}$ and $y_{t-2}$ chosen to match with selected values of the interest rate differential $\left(y_{t, 1}\right)$ and the exchange rate $\left(y_{t, 2}\right)$ series. The arrows point from $y_{t-2}$ to $y_{t-1}$ with these two points chosen as follows. Upper panel: $y_{t-2}$ and $y_{t-1}$ correspond to April 2000 and May 2000 (left), May 2000 and June 2000 (middle), and June 2000 and July 2000 (right). Lower panel: $y_{t-2}$ and $y_{t-1}$ correspond to October 2007 and November 2007 (left), November 2007 and December 2007 (middle), and December 2007 and January 2008 (right).

$\alpha_{1, t}$, and hence functions of $\left(y_{t-1,2}, y_{t-2,2}\right)$ (see equations (3) and (4)).

To better understand how the regime switches can occur in the GMVAR model (12), we examine the estimated mixing weights $\hat{\alpha}_{1, t}=P_{t-1}\left(s_{t, m}=1\right)$ graphically. As $\hat{\alpha}_{1, t}$ is a function of the four-dimensional vector $\boldsymbol{y}_{t-1}=\left(y_{t-1}, y_{t-2}\right)$, we consider two-dimensional projections as a function of $y_{t-1}$ with the arguments chosen to correspond to observed values of the two series during five successive months. Thus, the three figures in the upper panel and lower panel of Figure 2 are related to observed values of the interest rate differential and the exchange rate series during the periods from April 2000 to August 2000, and from October 2007 to February 2008, respectively. The former of these periods contains the big drop in the estimated mixing weights that lasts only a couple of months (see Figure 1, left panel), whereas the latter illustrates a more gradual shift in the estimated mixing weights.

The figure on the left in the upper panel of Figure 2 depicts contour plots of the mixing weight $\hat{\alpha}_{1, t}$ as a function of $y_{t-1}=\left(y_{t-1,1}, y_{t-1,2}\right)$ with the dot in the center of the 
contours being $y_{t-2}=\left(y_{t-2,1}, y_{t-2,2}\right)$ chosen to match with the observed values of the two series on April 2000. The arrow from this dot towards left points to $y_{t-1}$, the values of the two series on the next month, May 2000. Thus, the two dots give the four components of $\boldsymbol{y}_{t-1}$ that determine the value of the estimated mixing weight $\hat{\alpha}_{1, t}$ for June 2000, and, as can be seen from the figure, this value is below 0.1 , with the precise value being only 0.02. This is a large drop compared to the values of $\hat{\alpha}_{1, t}$ on April 2000 and May 2000 which are 0.91 and 0.82 , respectively. The contour plot in the middle of the upper panel shows the situation one month later. Thus, the dot in the center of this contour plot shows the components of $y_{t-2}$ on May 2000, with the arrow pointing to the dot showing the components of $y_{t-1}$ on June 2000. Together these two dots give the four components of $\boldsymbol{y}_{t-1}$ that determine the value of $\hat{\alpha}_{1, t}$ for July 2000, which is 0.49 . Similarly, the two dots on the right of the upper panel show the components of $\boldsymbol{y}_{t-1}$ that determine the value of $\hat{\alpha}_{1, t}$ for August 2000, which is 0.86 . Thus, the series has most likely visited the second regime (high mean, high variance regime) for only a month or two after May 2000 and then returned back to the first regime (low mean, low variance regime). What is interesting here is that the levels of both series have all the time been low, even below the estimated means of their stationary distributions which are -4.41 and 116.88 for the interest rate differential $\left(y_{t, 1}\right)$ and the exchange rate $\left(y_{t, 2}\right)$, respectively. This illustrates the fact mentioned in the introduction that the GMVAR model can allow for regime switches taking place in various combinations of high/low levels and high/low variability of the considered series.

Now consider the period from October 2007 to February 2008 depicted in the lower panel of Figure 2. Prior to this period, the estimated mixing weight $\hat{\alpha}_{1, t}$ takes values 0.73 and 0.80 in August and September of 2007. The two dots on the left of the lower panel of Figure 2 show the observations for October 2007 and November 2007 (the tip of the arrow being outside the largest contour) when the estimated mixing weights $\hat{\alpha}_{1, t}$ are 0.44 and 0.38, respectively, indicating a gradual decrease from the values in August and September. These two dots determine the value of $\hat{\alpha}_{1, t}$ on December 2007 which is 0.01. During the following two months the estimated mixing weights remain below 0.01 . These are depicted for December 2007 (the lower panel, left), January 2008 (the lower panel, middle), and February 2008 (the lower panel, right), respectively. As can be seen from Figure 1 (left panel) the estimated mixing weights remain very low till the end of the estimation period December 2009. 


\subsection{Model evaluation}

We next check the adequacy of the estimated GMVAR model. In mixture models, care is needed when residual-based diagnostics are used to evaluate fitted models, because empirical counterparts of the error terms $\varepsilon_{t}$ cannot be straightforwardly computed and, therefore, conventional residuals are not readily available. The reason for this is that the presence of the unobserved variables $s_{t, m}$ cannot be separated from the effect of $\varepsilon_{t}$ (see (1)). As in the univariate case in Kalliovirta et al. (2015), we use (multivariate) quantile residuals instead of conventional residuals in computing residual-based diagnostic tests. Tests for serial correlation, conditional heteroskedasticity, and non-normality in quantile residuals in multivariate models are developed by Kalliovirta and Saikkonen (2010) (see also Kalliovirta (2012) for similar tests in a univariate setting). ${ }^{5}$ Given the asymptotic results presented in Section 3.4, one can show that, under correct specification, the obtained $p$-values of these tests are asymptotically valid.

The results of the diagnostic tests based on multivariate quantile residuals are as follows. The test for normality has a $p$-value of 0.98 (this test is based on moments of multivariate quantile residuals, and under correct specification it is approximately $\chi_{4}^{2-}$ distributed). The test statistics for autocorrelation and conditional heteroskedasticity have $p$-values 0.12 and 0.46 , respectively (these tests are based on the first six serial covariances of the quantile residuals and on the first six serial covariances of squared quantile residuals, respectively, and under correct specification both of them are approximately $\chi_{24}^{2}$-distributed). These diagnostic tests show no indication of misspecification in the estimated GMVAR $(2,2)$ model (12). Graphical analyses related to these tests (including time series plots, auto and cross correlation functions, Q-Q plots, and kernel densities of the residuals; not reported here but available in the Supplementary Appendix) further support the adequacy the estimated GMVAR model.

\subsection{Forecasting comparisons}

We next compare the performance of the linear VAR model and the nonlinear GMVAR model in a forecasting exercise in which we also include several other models, although these models do not pass the residual-based diagnostics and some of them are found inferior to the GMVAR model by information criteria. As competing nonlinear multivariate models, we include the MVAR model of Fong et al. (2007), the C-MSTAR model

\footnotetext{
${ }^{5}$ When the null hypothesis is a linear $\operatorname{VAR}(p)$ model our test for conditional heteroskedasticity appears very similar to the general portmanteau-type test Dueker et al. (2011) use to test for nonlinearity.
} 
of Dueker et al. (2011), and the (time-homogeneous) MS-VAR model (see, e.g., Krolzig (1997)). Furthermore, we also consider a restricted version of the $\operatorname{GMVAR}(2,2)$ model, GMVAR $^{\mathrm{r}}$, in which the off-diagonal elements of the autoregressive parameters are restricted to zero, and all other parameters are estimated freely (see (12) and the discussion in Section 4.2). A number of other models were also tried; for brevity all results and discussion regarding these is relegated to the Supplementary Appendix.

The parameters of all the models considered are estimated using maximum likelihood and observations from January 1989 to December 2009 (the parameter estimates, values of information criteria, and results of appropriate diagnostic tests are available in the Supplementary Appendix). We use a fixed forecasting scheme so that all the forecasts are based on these parameter estimates. The date of forecasting ranges from December 2009 till November 2013, and for each date of forecasting, forecasts are computed for all the subsequent periods up until December 2013. Assuming correct specification, optimal one-step-ahead forecasts (in mean squared sense and ignoring estimation errors) are straightforward to compute with each model because explicit formulas are available for the conditional expectation (see (3)). However, computing multi-step forecasts is more complicated for mixture models because explicit formulas are very difficult to obtain. Therefore, we use the common practice and resort to simulation-based methods (see, e.g., Dueker et al. (2007, Sec. 4.2), Teräsvirta, Tjøstheim, and Granger (2010, Ch. 14), and the references therein). For mixture models multi-step forecasts are computed as follows. Using each of the estimated mixture models and initial values known at the date of forecasting, we simulate 500,000 realizations and treat the mean of these realizations as a point forecast. We repeat this for all forecast horizons up until December 2013. This results in a total of 48 one-step forecasts, 47 two-step forecasts, . ., 39 ten-step forecasts (as well as forecasts for longer horizons which we discard).

We examine forecast accuracy of point forecasts for the two (univariate) component series separately as well as for the bivariate system as a whole. Forecast accuracy is measured using mean squared prediction error (MSPE) for the forecasts of the component series, and using the determinant of the covariance matrix of the forecast error vectors in the multivariate case. The results are presented in Figure 3: The left panel presents MSPEs when forecasting interest rates, middle panel the MSPEs for the exchange rate series, and the right panel the determinant of the covariance matrix of the forecast error vectors for the bivariate system. Forecast accuracy of the models is presented relative to the GMVAR model: The straight line (at 100) represents the GMVAR model, whereas the other lines represent the size of the forecast error made relative to the GMVAR model (for 

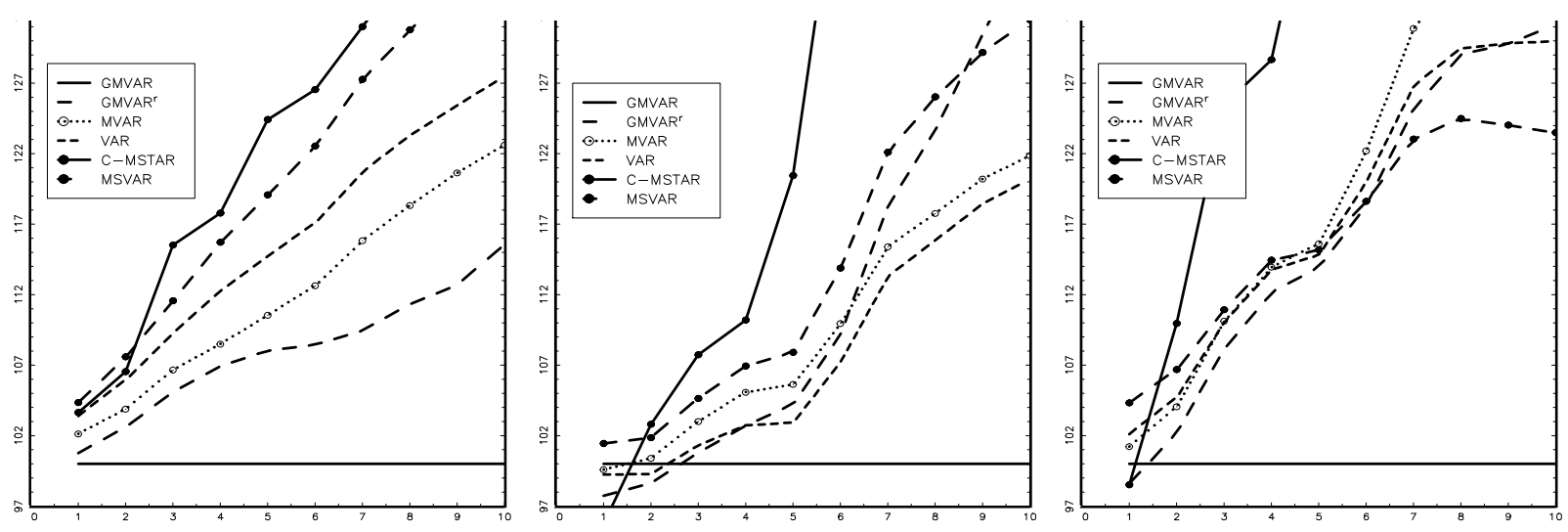

Figure 3: Relative forecast accuracies in the interest rate series (left) and the exchange rate series (middle) using mean squared prediction error (MSPE), and the determinant of the covariance matrix of forecast error vectors (right).

instance, a value of 109 in the figure is to be interpreted as an MSPE (or a determinant of the covariance matrix of the forecast error vector) $9 \%$ larger than for the GMVAR model).

As far as forecasting the interest rate series is concerned, the relative merits of the competing models are clear: The most accurate forecasts are produced by the GMVAR model, followed by the restricted GMVAR model, the MVAR model, the VAR model, and finally the C-MSTAR and MS-VAR models. Except for the last two models, this ranking remains the same across all the considered forecast horizons (1-step, .., 10step). The results are less clear when forecasts of the exchange rate series are compared. All six models are more or less equally good in 1-step and 2-step ahead forecasting (the best 1-step ahead forecasts are produced by the C-MSTAR model and the best 2-step ahead forecasts by the GMVAR ${ }^{\mathrm{r}}$ model, but overall the differences are small). For longer forecast horizons the GMVAR model again outperforms its competitors. The rightmost panel of Figure 3 summarizes the forecast accuracy of the models using the determinant of the covariance matrix of the forecast error vectors. The C-MSTAR model narrowly outperforms the other models in 1-step ahead forecasting, but performs less well at longer forecast horizons. The GMVAR model produces the most accurate forecasts for all forecast horizons larger than 1 . The other models perform more or less equally well, with their ranking depending on the forecast horizon. Overall, we can conclude that the GMVAR model performs quite well in comparison with its competitors. Details of the forecasting accuracy of the GMVAR $(2,2)$ model can be found in Section A1.4 of the Supplementary Appendix. Here we only note that the forecast accuracy was best in one-step-ahead 
Table 1: The percentage shares of observations that belong to the $80 \%$ and $90 \%$ prediction intervals based on the distribution of 500,000 one-step ahead simulated forecasts.

\begin{tabular}{lccccc}
\hline \hline & \multicolumn{2}{c}{ Interest rate } & & \multicolumn{2}{c}{ Exchange rate } \\
& $80 \%$ & $90 \%$ & & $80 \%$ & $90 \%$ \\
\cline { 2 - 3 } \cline { 5 - 6 } GMVAR & 79 & 92 & & 81 & 90 \\
GMVAR & 79 & 90 & & 81 & 90 \\
VAR & 75 & 79 & & 81 & 90 \\
MVAR & 71 & 79 & & 77 & 90 \\
C-MSTAR & 71 & 79 & & 79 & 92 \\
MS-VAR & 71 & 79 & & 79 & 85 \\
\hline \hline
\end{tabular}

prediction and, as expected, steadily deteriorated with the forecast horizon. The root MSPEs for 1-step, 2-step, and 4-step forecasts are 2.30, 3.62, and 5.27 for the interest rate series and 3.09, 4.82, and 6.57 for the exchange rate series (the standard deviations of the interest rate series and exchange rate series are 12.53 and 15.56, respectively).

We also examined one-step ahead prediction intervals produced by the six competing models. Table 1 presents the percentage shares of observations that belong to the $80 \%$ and $90 \%$ prediction intervals based on the distribution of 500,000 simulated one-step ahead forecasts. It is seen that for the interest rate series the empirical coverage rates of the GMVAR and GMVAR ${ }^{\mathrm{r}}$ based prediction intervals are closer to the nominal $80 \%$ and $90 \%$ levels than the ones obtained with the other models, whereas for the exchange rate series the differences are small. Christoffersen's (1998) tests indicate that for the interest rate series the $90 \%$ prediction intervals produced by the VAR, MVAR, C-MSTAR, and MS-VAR models have incorrect coverage probabilities, whereas all the other prediction intervals in Table 1 have correct coverage probabilities (for details, see Section A1.6 of the Supplementary Appendix). This better predictive accuracy may be partly explained by the particular mixing weights used in the GMVAR models.

\section{Conclusion}

This paper introduces a new mixture VAR model referred to as the Gaussian mixture vector autoregressive (GMVAR) model. Due to the particular specification of the mixing weights the GMVAR model has a clear probability structure with simple conditions ensuring stationarity and ergodicity. Building on these properties of the GMVAR model, the paper develops an asymptotic theory of maximum likelihood estimation establishing 
the consistency and asymptotic normality of the ML estimator.

In addition to theoretical attractiveness, an appealing feature of the mixing weights employed in the GMVAR model is that they depend on past values of the series in a way which enables the researcher to associate different regimes of the model to different states of economy. In this respect the GMVAR model is very flexible being capable of allowing for regime switches that take place, for instance, in periods of high/low levels of the considered series, or in periods of high/low variability, or high/low temporal dependence, or combinations of all of these.

The practical use of the new model is illustrated by a bivariate example on exchange rate and interest rate data. A GMVAR model with two economically meaningful regimes is found to provide a good in-sample fit and good forecasting power in comparison to considered alternatives. 


\section{Technical Appendix}

Proof of Theorem 1. We first note some properties of the stationary auxiliary vector autoregressions $\nu_{m, t}$. Denoting $\boldsymbol{\nu}_{m, t}^{+}=\left(\nu_{m, t}, \boldsymbol{\nu}_{m, t-1}\right)$ and $\mathbf{1}_{p+1}=(1, \ldots, 1)((p+1) \times 1)$, it is seen that $\boldsymbol{\nu}_{m, t}^{+}$follows the $d(p+1)$-dimensional multivariate normal distribution with density

$$
\begin{aligned}
\mathrm{n}_{d(p+1)}\left(\boldsymbol{\nu}_{m, t}^{+} ; \boldsymbol{\vartheta}_{m}\right)= & (2 \pi)^{-d(p+1) / 2} \operatorname{det}\left(\boldsymbol{\Sigma}_{m, p+1}\right)^{-1 / 2} \\
& \times \exp \left\{-\frac{1}{2}\left(\boldsymbol{\nu}_{m, t}^{+}-\mathbf{1}_{p+1} \otimes \mu_{m}\right)^{\prime} \boldsymbol{\Sigma}_{m, p+1}^{-1}\left(\boldsymbol{\nu}_{m, t}^{+}-\mathbf{1}_{p+1} \otimes \mu_{m}\right)\right\},
\end{aligned}
$$

where the matrices $\boldsymbol{\Sigma}_{m, p+1}, m=1, \ldots, M$, have the usual symmetric block Toeplitz form. This joint density can be decomposed as

$$
\mathrm{n}_{d(p+1)}\left(\boldsymbol{\nu}_{m, t}^{+} ; \boldsymbol{\vartheta}_{m}\right)=\mathrm{n}_{d}\left(\nu_{m, t} \mid \boldsymbol{\nu}_{m, t-1} ; \boldsymbol{\vartheta}_{m}\right) \mathrm{n}_{d p}\left(\boldsymbol{\nu}_{m, t-1} ; \boldsymbol{\vartheta}_{m}\right)
$$

where the normality of the two densities on the right-hand side follows from properties of the multivariate normal distribution (see, e.g., Anderson (2003, Thms 2.4.3 and 2.5.1)). Moreover, $\mathrm{n}_{d p}\left(\cdot ; \boldsymbol{\vartheta}_{m}\right)$ clearly has the form given in (6), and making use of the Yule-Walker equations for VAR processes (see, e.g., Reinsel (1997, Sec. 2.2.2)) together with the identity $\mu_{m}=A_{m}^{-1}(1) \phi_{m, 0}$, it can be seen that

$$
\mathrm{n}_{d}\left(\nu_{m, t} \mid \boldsymbol{\nu}_{m, t-1} ; \boldsymbol{\vartheta}_{m}\right)=(2 \pi)^{-d / 2} \operatorname{det}\left(\Omega_{m}\right)^{-1 / 2} \exp \left\{-\frac{1}{2}\left(\nu_{m, t}-\mu_{m, t}\right)^{\prime} \Omega_{m}^{-1}\left(\nu_{m, t}-\mu_{m, t}\right)\right\}
$$

where $\mu_{m, t}$ is defined in Condition 1(a).

The rest of the proof makes use of the theory of Markov chains (for the employed concepts, see Meyn and Tweedie (2009)). As was noted in the discussion preceding the theorem, $\boldsymbol{y}_{t}$ is a Markov chain on $\mathbb{R}^{d p}$. Now let $\boldsymbol{y}_{0}=\left(y_{0}, \ldots, y_{-p+1}\right)$ be a random vector whose distribution has the density $f\left(\boldsymbol{y}_{0} ; \boldsymbol{\theta}\right)=\sum_{m=1}^{M} \alpha_{m} \mathrm{n}_{d p}\left(\boldsymbol{y}_{0} ; \boldsymbol{\vartheta}_{m}\right)$. According to (2), (7), (13), and (14), the conditional density of $y_{1}$ given $\boldsymbol{y}_{0}$ is

$$
\begin{aligned}
f\left(y_{1} \mid \boldsymbol{y}_{0} ; \boldsymbol{\theta}\right) & =\sum_{m=1}^{M} \frac{\alpha_{m}}{\sum_{n=1}^{M} \alpha_{n} \mathrm{n}_{d p}\left(\boldsymbol{y}_{0} ; \boldsymbol{\vartheta}_{n}\right)} \mathrm{n}_{d p}\left(\boldsymbol{y}_{0} ; \boldsymbol{\vartheta}_{m}\right) \mathrm{n}_{d}\left(y_{1} \mid \boldsymbol{y}_{0} ; \boldsymbol{\vartheta}_{m}\right) \\
& =\sum_{m=1}^{M} \frac{\alpha_{m}}{\sum_{n=1}^{M} \alpha_{n} \mathrm{n}_{d p}\left(\boldsymbol{y}_{0} ; \boldsymbol{\vartheta}_{n}\right)} \mathrm{n}_{d(p+1)}\left(\left(y_{1}, \boldsymbol{y}_{0}\right) ; \boldsymbol{\vartheta}_{m}\right) .
\end{aligned}
$$

It thus follows that the density of $\left(y_{1}, \boldsymbol{y}_{0}\right)=\left(y_{1}, y_{0}, \ldots, y_{-p+1}\right)$ is

$$
f\left(\left(y_{1}, \boldsymbol{y}_{0}\right) ; \boldsymbol{\theta}\right)=\sum_{m=1}^{M} \alpha_{m} \mathrm{n}_{d(p+1)}\left(\left(y_{1}, \boldsymbol{y}_{0}\right) ; \boldsymbol{\vartheta}_{m}\right)
$$


Integrating $y_{-p+1}$ away it follows that the density of $\boldsymbol{y}_{1}$ is $f\left(\boldsymbol{y}_{1} ; \boldsymbol{\theta}\right)=\sum_{m=1}^{M} \alpha_{m} \mathrm{n}_{d p}\left(\boldsymbol{y}_{1} ; \boldsymbol{\vartheta}_{m}\right)$. Therefore, $\boldsymbol{y}_{0}$ and $\boldsymbol{y}_{1}$ are identically distributed. As already noted, $\left\{\boldsymbol{y}_{t}\right\}_{t=1}^{\infty}$ is a (time homogeneous) Markov chain, and hence we can conclude that $\left\{\boldsymbol{y}_{t}\right\}_{t=1}^{\infty}$ has a stationary distribution $\pi_{\boldsymbol{y}}(\cdot)$, say, characterized by the density $f(\cdot ; \boldsymbol{\theta})=\sum_{m=1}^{M} \alpha_{m} \mathrm{n}_{d p}\left(\cdot ; \boldsymbol{\vartheta}_{m}\right)($ cf. Meyn and Tweedie (2009, pp. 230-231)). As a mixture of multivariate normal distributions, all moments of $\boldsymbol{y}_{t}$ are finite.

It remains to establish ergodicity. To this end, let $P_{\boldsymbol{y}}^{p}(\boldsymbol{y}, \cdot)=\operatorname{Pr}\left(\boldsymbol{y}_{p} \mid \boldsymbol{y}_{0}=\boldsymbol{y}\right)$ signify the $p$-step transition probability measure of $\boldsymbol{y}_{t}$. It is straightforward to check that $P_{\boldsymbol{y}}^{p}(\boldsymbol{y}, \cdot)$ has a density given by

$$
f\left(\boldsymbol{y}_{p} \mid \boldsymbol{y}_{0} ; \boldsymbol{\theta}\right)=\prod_{t=1}^{p} f\left(y_{t} \mid \boldsymbol{y}_{t-1} ; \boldsymbol{\theta}\right)=\prod_{t=1}^{p} \sum_{m=1}^{M} \alpha_{m, t} \mathrm{n}_{d}\left(y_{t} \mid \boldsymbol{y}_{t-1} ; \boldsymbol{\vartheta}_{m}\right) .
$$

The last expression makes clear that $f\left(\boldsymbol{y}_{p} \mid \boldsymbol{y}_{0} ; \boldsymbol{\theta}\right)>0$ for all $\boldsymbol{y}_{p} \in \mathbb{R}^{d p}$ and all $\boldsymbol{y}_{0} \in \mathbb{R}^{d p}$ so that, from every initial state $\boldsymbol{y}_{0}=\boldsymbol{y}\left(\in \mathbb{R}^{d p}\right)$, the chain $\boldsymbol{y}_{t}$ can in $p$ steps reach any set of the state space $\mathbb{R}^{d p}$ with positive Lebesgue measure. Using the definitions of irreducibility and aperiodicity we can therefore conclude that the chain $\boldsymbol{y}_{t}$ is irreducible and aperiodic (see Meyn and Tweedie (2009, Chapters 4.3 and 5.4)). Moreover, also the $p$-step transition probability measure $P_{\boldsymbol{y}}^{p}(\boldsymbol{y}, \cdot)$ is irreducible, aperiodic, and has $\pi_{\boldsymbol{y}}$ as its stationary distribution (see Meyn and Tweedie, 2009, Theorem 10.4.5).

A further consequence of the preceding discussion is that the $p$-step transition probability measure $P_{\boldsymbol{y}}^{p}(\boldsymbol{y}, \cdot)$ is equivalent to the Lebesgue measure on $\mathbb{R}^{d p}$ for all $\boldsymbol{y} \in \mathbb{R}^{d p}$. As the stationary probability measure $\pi_{\boldsymbol{y}}(\cdot)$ also has a (Lebesgue) density positive everywhere in $\mathbb{R}^{d p}$ it is likewise equivalent to the Lebesgue measure on $\mathbb{R}^{d p}$. Consequently, the $p$-step transition probability measure $P_{\boldsymbol{y}}^{p}(\boldsymbol{y}, \cdot)$ is absolutely continuous with respect to the stationary probability measure $\pi_{\boldsymbol{y}}(\cdot)$ for all $\boldsymbol{y} \in \mathbb{R}^{d p}$.

To complete the proof, we now use the preceding facts and conclude from Theorem 1 and Corollary 1 of Tierney (1994) that $\left\|P_{\boldsymbol{y}}^{p n}(\boldsymbol{y}, \cdot)-\pi_{\boldsymbol{y}}(\cdot)\right\| \rightarrow 0$ as $n \rightarrow \infty$ for all $\boldsymbol{y} \in \mathbb{R}^{d p}$, where $\|\cdot\|$ signifies the total variation norm of probability measures. Now, by Proposition 13.3.2 of Meyn and Tweedie (2009), also $\left\|P_{\boldsymbol{y}}^{n}(\boldsymbol{y}, \cdot)-\pi_{\boldsymbol{y}}(\cdot)\right\| \rightarrow 0$ as $n \rightarrow \infty$ for all $\boldsymbol{y} \in \mathbb{R}^{d p}$ (as the total variation norm is non-increasing in $n$ ). Hence, $\boldsymbol{y}_{t}$ is ergodic in the sense of Meyn and Tweedie (2009, Ch. 13).

Proof of Theorem 2. We consider the conditional ML estimator obtained by maximizing $L_{T}(\boldsymbol{\theta})=T^{-1} \sum_{t=1}^{T} l_{t}(\boldsymbol{\theta})$ (it is easy to verify that the conditional and exact $\mathrm{ML}$ estimators are asymptotically equivalent), and assume stationary initial values (the same 
result can be obtained without assuming this). Assumption 1 together with the continuity of the log-likelihood function $L_{T}(\boldsymbol{\theta})$ implies the existence of a measurable maximizer $\hat{\boldsymbol{\theta}}_{T}$ of $L_{T}(\boldsymbol{\theta})$ (see, e.g., Pötscher and Prucha, 1991, Lemma 3.4). For the strong consistency it suffices to show that the (conditional) log-likelihood obeys a uniform strong law of large numbers, that is, $\sup _{\boldsymbol{\theta} \in \Theta}\left|L_{T}(\boldsymbol{\theta})-E\left[L_{T}(\boldsymbol{\theta})\right]\right| \rightarrow 0$ a.s. as $T \rightarrow \infty$, and that an identification condition (to be specified below) holds. For the former, as the initial values are drawn from the stationary distribution, the process is stationary and ergodic and $E\left[L_{T}(\boldsymbol{\theta})\right]=E\left[l_{t}(\boldsymbol{\theta})\right]$, and it thus suffices to show that $E\left[\sup _{\boldsymbol{\theta} \in \Theta}\left|l_{t}(\boldsymbol{\theta})\right|\right]<\infty$ (see, e.g., Ranga Rao (1962)). To this end, Assumption 1 implies that $\delta \leq \operatorname{det}\left(\Omega_{m}\right) \leq \Delta$ and $\delta \leq \alpha_{m} \leq 1-\delta$ for some $0<\delta<1$ and $\Delta<\infty, m=1, \ldots, M$. Now, because $0 \leq \alpha_{m, t} \leq 1$ and $\operatorname{det}\left(\Omega_{m}\right) \geq \delta$ for all $m=1, \ldots, M$, and because the exponential function is bounded from above by unity on the non-positive real axis (and $\Omega_{m}$ is positive definite), we can find a $\bar{C}<\infty$ such that

$$
l_{t}(\boldsymbol{\theta})=\log \left(\sum_{m=1}^{M} \alpha_{m, t}(2 \pi)^{-d / 2} \operatorname{det}\left(\Omega_{m}\right)^{-1 / 2} \exp \left\{-\frac{1}{2}\left(y_{t}-\mu_{m, t}\right)^{\prime} \Omega_{m}^{-1}\left(y_{t}-\mu_{m, t}\right)\right\}\right) \leq \bar{C}
$$

for all $\boldsymbol{\theta} \in \Theta$. On the other hand, making use of properties of the trace operator and the fact that $\Theta$ is compact it can be seen that

$$
\left(y_{t}-\mu_{m, t}\right)^{\prime} \Omega_{m}^{-1}\left(y_{t}-\mu_{m, t}\right) \leq c_{1}\left(1+y_{t}^{\prime} y_{t}+\boldsymbol{y}_{t-1}^{\prime} \boldsymbol{y}_{t-1}\right), \quad m=1, \ldots, M,
$$

for all $\boldsymbol{\theta} \in \Theta$ with some finite $c_{1}$. As $\operatorname{det}\left(\Omega_{m}\right) \leq \Delta$ for all $m=1, \ldots, M$, we thus get

$$
\mathrm{n}_{d}\left(y_{t} \mid \boldsymbol{y}_{t-1} ; \boldsymbol{\vartheta}_{m}\right) \geq(2 \pi)^{-d / 2} \Delta^{-1 / 2} \exp \left\{-\frac{1}{2} c_{1}\left(1+y_{t}^{\prime} y_{t}+\boldsymbol{y}_{t-1}^{\prime} \boldsymbol{y}_{t-1}\right)\right\}
$$

for all $\boldsymbol{\theta} \in \Theta$ and all $m=1, \ldots, M$. Therefore, as $\sum_{m=1}^{M} \alpha_{m, t}=1$,

$$
\begin{aligned}
l_{t}(\boldsymbol{\theta}) & =\log \left(\sum_{m=1}^{M} \alpha_{m, t} \mathrm{n}_{d}\left(y_{t} \mid \boldsymbol{y}_{t-1} ; \boldsymbol{\vartheta}_{m}\right)\right) \\
& \geq-\frac{d}{2} \log (2 \pi)-\frac{1}{2} \log (\Delta)-\frac{1}{2} c_{1}\left(1+y_{t}^{\prime} y_{t}+\boldsymbol{y}_{t-1}^{\prime} \boldsymbol{y}_{t-1}\right)
\end{aligned}
$$

for all $\boldsymbol{\theta} \in \Theta$. Defining $\underline{C}<\infty$ suitably and combining with the result obtained above, $-\underline{C}\left(1+y_{t}^{\prime} y_{t}+\boldsymbol{y}_{t-1}^{\prime} \boldsymbol{y}_{t-1}\right) \leq l_{t}(\boldsymbol{\theta}) \leq \bar{C}$, from which $E\left[\sup _{\boldsymbol{\theta} \in \Theta}\left|l_{t}(\boldsymbol{\theta})\right|\right]<\infty$ follows because $E\left[y_{t}^{\prime} y_{t}+\boldsymbol{y}_{t-1}^{\prime} \boldsymbol{y}_{t-1}\right]<\infty$.

Concerning the identification condition, we next establish that $E\left[l_{t}(\boldsymbol{\theta})\right] \leq E\left[l_{t}\left(\boldsymbol{\theta}_{0}\right)\right]$, and $E\left[l_{t}(\boldsymbol{\theta})\right]=E\left[l_{t}\left(\boldsymbol{\theta}_{0}\right)\right]$ implies $\boldsymbol{\vartheta}_{m}=\boldsymbol{\vartheta}_{\tau(m), 0}$ and $\alpha_{m}=\alpha_{\tau(m), 0}(m=1, \ldots, M)$ for some permutation $\{\tau(1), \ldots, \tau(M)\}$ of $\{1, \ldots, M\}$. In light of $(11)$, this implies that $\boldsymbol{\theta}=\boldsymbol{\theta}_{0}$. 
Choose an arbitrary $\boldsymbol{\theta}$ and consider the difference $E\left[l_{t}(\boldsymbol{\theta})\right]-E\left[l_{t}\left(\boldsymbol{\theta}_{0}\right)\right]$. First note that the density of $\left(y_{t}, \boldsymbol{y}_{t-1}\right)$ can be written as

$$
\sum_{m=1}^{M} \alpha_{m, 0} \mathrm{n}_{d(p+1)}\left(\left(y_{t}, \boldsymbol{y}_{t-1}\right) ; \boldsymbol{\vartheta}_{m, 0}\right)=\sum_{n=1}^{M} \alpha_{n, 0} \mathrm{n}_{d p}\left(\boldsymbol{y}_{t-1} ; \boldsymbol{\vartheta}_{n, 0}\right) \sum_{m=1}^{M} \alpha_{m, 0, t} \mathrm{n}_{d}\left(y_{t} \mid \boldsymbol{y}_{t-1} ; \boldsymbol{\vartheta}_{m, 0}\right)
$$

so that $E\left[l_{t}(\boldsymbol{\theta})\right]-E\left[l_{t}\left(\boldsymbol{\theta}_{0}\right)\right]$ can be written as

$$
\begin{aligned}
& E\left[l_{t}(\boldsymbol{\theta})\right]-E\left[l_{t}\left(\boldsymbol{\theta}_{0}\right)\right] \\
= & \iint \sum_{m=1}^{M} \alpha_{m, 0} \mathrm{n}_{d(p+1)}\left((y, \boldsymbol{y}) ; \boldsymbol{\vartheta}_{m, 0}\right) \log \left(\frac{\sum_{m=1}^{M} \alpha_{m, t} \mathrm{n}_{d}\left(y \mid \boldsymbol{y} ; \boldsymbol{\vartheta}_{m}\right)}{\sum_{m=1}^{M} \alpha_{m, 0, t} \mathrm{n}_{d}\left(y \mid \boldsymbol{y} ; \boldsymbol{\vartheta}_{m, 0}\right)}\right) d y d \boldsymbol{y} \\
= & \int \sum_{n=1}^{M} \alpha_{n, 0} \mathrm{n}_{d p}\left(\boldsymbol{y} ; \boldsymbol{\vartheta}_{n, 0}\right)\left[\int \sum_{m=1}^{M} \alpha_{m, 0, t} \mathrm{n}_{d}\left(y \mid \boldsymbol{y} ; \boldsymbol{\vartheta}_{m, 0}\right) \log \left(\frac{\sum_{m=1}^{M} \alpha_{m, t} \mathrm{n}_{d}\left(y \mid \boldsymbol{y} ; \boldsymbol{\vartheta}_{m}\right)}{\sum_{m=1}^{M} \alpha_{m, 0, t} \mathrm{n}_{d}\left(y \mid \boldsymbol{y} ; \boldsymbol{\vartheta}_{m, 0}\right)}\right) d y\right] d \boldsymbol{y} .
\end{aligned}
$$

(Here and in what follows, for conciseness we continue using the notation $\alpha_{m, t}$ although now $\boldsymbol{y}_{t-1}$ in (7) is replaced by $\boldsymbol{y}$.) The inner integral is, for every fixed $\boldsymbol{y}$, the (negative) of the Kullback-Leibler divergence between the two mixture densities $\sum_{m=1}^{M} \alpha_{m, t} \mathbf{n}_{d}\left(y \mid \boldsymbol{y} ; \boldsymbol{\vartheta}_{m}\right)$ and $\sum_{m=1}^{M} \alpha_{m, 0, t} \mathbf{n}_{d}\left(y \mid \boldsymbol{y} ; \boldsymbol{\vartheta}_{m, 0}\right)$. Therefore, $E\left[l_{t}(\boldsymbol{\theta})\right]-E\left[l_{t}\left(\boldsymbol{\theta}_{0}\right)\right] \leq 0$, with equality if and only if for almost all $(y, \boldsymbol{y})$,

$$
\sum_{m=1}^{M} \alpha_{m, t} \mathrm{n}_{d}\left(y \mid \boldsymbol{y} ; \boldsymbol{\vartheta}_{m}\right)=\sum_{m=1}^{M} \alpha_{m, 0, t} \mathrm{n}_{d}\left(y \mid \boldsymbol{y} ; \boldsymbol{\vartheta}_{m, 0}\right) .
$$

For each fixed $\boldsymbol{y}$ at a time, the mixing weights are constants, and we may apply the results on identification of finite mixtures of Gaussian distributions in Yakowitz and Spragins (1968, Proposition 2) (see also (11)). Consequently, for each fixed $\boldsymbol{y}$ at a time, there exists a permutation $\{\tau(1), \ldots, \tau(M)\}$ of $\{1, \ldots, M\}$ (where this permutation may depend on $\boldsymbol{y})$ such that

$$
\alpha_{m, t}=\alpha_{\tau(m), 0, t} \text { and } \mathbf{n}_{d}\left(y \mid \boldsymbol{y} ; \boldsymbol{\vartheta}_{m}\right)=\mathrm{n}_{d}\left(y \mid \boldsymbol{y} ; \boldsymbol{\vartheta}_{\tau(m), 0}\right) \text { for almost all } y(m=1, \ldots, M) \text {. }
$$

The number of possible permutations being finite $(M !)$, this induces a finite partition of $\mathbb{R}^{d p}$ where the elements $\boldsymbol{y}$ of each partition correspond to the same permutation. At least one of these partitions, say $A \subset \mathbb{R}^{d p}$, must have positive Lebesque measure. Thus, we may conclude that (15) holds for all fixed $\boldsymbol{y} \in A$ with some specific permutation $\{\tau(1), \ldots, \tau(M)\}$ of $\{1, \ldots, M\}$.

The latter condition in (15) means that (denoting $A_{m}=\left[A_{m, 1}: \cdots: A_{m, p}\right](d \times d p)$ and $A_{m, 0}$ similarly using the true parameter values)

$$
\begin{aligned}
& \operatorname{det}\left(\Omega_{m}\right)^{-1 / 2} \exp \left\{-\frac{1}{2}\left(y-\phi_{m, 0}-A_{m} \boldsymbol{y}\right)^{\prime} \Omega_{m}^{-1}\left(y-\phi_{m, 0}-A_{m} \boldsymbol{y}\right)\right\} \\
= & \operatorname{det}\left(\Omega_{\tau(m), 0}\right)^{-1 / 2} \exp \left\{-\frac{1}{2}\left(y-\phi_{\tau(m), 0,0}-A_{\tau(m), 0} \boldsymbol{y}\right)^{\prime} \Omega_{\tau(m), 0}^{-1}\left(y-\phi_{\tau(m), 0,0}-A_{\tau(m), 0} \boldsymbol{y}\right)\right\}
\end{aligned}
$$


for $m=1, \ldots, M$, almost all $y \in \mathbb{R}^{d}$, and all $\boldsymbol{y} \in A$. This implies that $\Omega_{m}=\Omega_{\tau(m), 0}$ and $\phi_{m, 0}-\phi_{\tau(m), 0,0}+\left(A_{m}-A_{\tau(m), 0}\right) \boldsymbol{y}=0, m=1, \ldots, M$, for all $\boldsymbol{y} \in A$. The latter equalities imply either that $\left(\phi_{m, 0}, \phi_{m}\right)=\left(\phi_{\tau(m), 0,0}, \phi_{\tau(m), 0}\right)$, or that $\boldsymbol{y}$ takes values only on a $d(p-1)$-dimensional hyperplane. As $A$ has positive Lebesgue measure, the latter is not possible. Therefore $\boldsymbol{\vartheta}_{m}=\left(\phi_{m, 0}, \boldsymbol{\phi}_{m}, \sigma_{m}\right)=\left(\phi_{\tau(m), 0,0}, \phi_{\tau(m), 0}, \sigma_{\tau(m), 0}\right)=\boldsymbol{\vartheta}_{\tau(m), 0}$ $(m=1, \ldots, M)$.

Now, the former condition in (15) means that

$$
\frac{\alpha_{m} \mathrm{n}_{d p}\left(\boldsymbol{y} ; \boldsymbol{\vartheta}_{m}\right)}{\sum_{n=1}^{M} \alpha_{n} \mathrm{n}_{d p}\left(\boldsymbol{y} ; \boldsymbol{\vartheta}_{n}\right)}=\frac{\alpha_{\tau(m), 0} \mathrm{n}_{d p}\left(\boldsymbol{y} ; \boldsymbol{\vartheta}_{\tau(m), 0}\right)}{\sum_{n=1}^{M} \alpha_{\tau(n), 0} \mathrm{n}_{d p}\left(\boldsymbol{y} ; \boldsymbol{\vartheta}_{\tau(n), 0}\right)}, \quad m=1, \ldots, M
$$

for all $\boldsymbol{y} \in A$. Cancelling $\mathrm{n}_{d p}\left(\boldsymbol{y} ; \boldsymbol{\vartheta}_{m}\right)=\mathrm{n}_{d p}\left(\boldsymbol{y} ; \boldsymbol{\vartheta}_{\tau(m), 0}\right)$ and rearranging,

$$
\frac{\alpha_{m}}{\alpha_{\tau(m), 0}}=\frac{\sum_{n=1}^{M} \alpha_{n} \mathrm{n}_{d p}\left(\boldsymbol{y} ; \boldsymbol{\vartheta}_{n}\right)}{\sum_{n=1}^{M} \alpha_{\tau(n), 0} \mathrm{n}_{d p}\left(\boldsymbol{y} ; \boldsymbol{\vartheta}_{\tau(n), 0}\right)}, \quad m=1, \ldots, M
$$

for all $\boldsymbol{y} \in A$. As the right hand side does not depend on $m$, we obtain $\alpha_{1} / \alpha_{\tau(1), 0}=\cdots=$ $\alpha_{M} / \alpha_{\tau(M), 0}$, which implies $\alpha_{m}=\alpha_{\tau(m), 0}(m=1, \ldots, M)$. This completes the proof.

Proof of Theorem 3. Let $\Theta_{0}$ be a compact convex set contained in the interior of $\Theta$ that has $\boldsymbol{\theta}_{0}$ as an interior point, and introduce the notation $l_{\boldsymbol{\theta}, t}(\boldsymbol{\theta})=\partial l_{t}(\boldsymbol{\theta}) / \partial \boldsymbol{\theta}, l_{\boldsymbol{\theta} \boldsymbol{\theta}, t}(\boldsymbol{\theta})=$ $\partial^{2} l_{t}(\boldsymbol{\theta}) / \partial \boldsymbol{\theta} \partial \boldsymbol{\theta}^{\prime}, L_{\boldsymbol{\theta}, T}(\boldsymbol{\theta})=\partial L_{T}(\boldsymbol{\theta}) / \partial \boldsymbol{\theta}$, and $L_{\boldsymbol{\theta} \boldsymbol{\theta}, T}(\boldsymbol{\theta})=\partial^{2} L_{T}(\boldsymbol{\theta}) / \partial \boldsymbol{\theta} \partial \boldsymbol{\theta}^{\prime}$. Expressions of $l_{\boldsymbol{\theta}, t}(\boldsymbol{\theta})$ and $l_{\boldsymbol{\theta} \boldsymbol{\theta}, t}(\boldsymbol{\theta})$ in Lemmas 1 and 2 below make clear that $l_{t}(\boldsymbol{\theta})$ is twice continuously differentiable on $\Theta_{0}$. A standard mean value expansion of the score vector $L_{\boldsymbol{\theta}, T}(\boldsymbol{\theta})$ yields

$$
T^{1 / 2} L_{\boldsymbol{\theta}, T}\left(\hat{\boldsymbol{\theta}}_{T}\right)=T^{1 / 2} L_{\boldsymbol{\theta}, T}\left(\boldsymbol{\theta}_{0}\right)+\dot{L}_{\boldsymbol{\theta} \boldsymbol{\theta}, T} T^{1 / 2}\left(\hat{\boldsymbol{\theta}}_{T}-\boldsymbol{\theta}_{0}\right) \text { a.s. },
$$

where $\dot{L}_{\boldsymbol{\theta} \boldsymbol{\theta}, T}$ signifies the matrix $L_{\boldsymbol{\theta} \boldsymbol{\theta}, T}(\boldsymbol{\theta})$ with each row evaluated at an intermediate point $\dot{\boldsymbol{\theta}}_{i, T}(i=1, \ldots, \operatorname{dim} \boldsymbol{\theta})$ lying between $\hat{\boldsymbol{\theta}}_{T}$ and $\boldsymbol{\theta}_{0}$. By Theorem $2, \hat{\boldsymbol{\theta}}_{T} \rightarrow \boldsymbol{\theta}_{0}$ a.s., so that $\dot{\boldsymbol{\theta}}_{i, T} \rightarrow \boldsymbol{\theta}_{0}$ a.s. as $T \rightarrow \infty(i=1, \ldots, \operatorname{dim} \boldsymbol{\theta})$ which, together with the uniform convergence result for $L_{\boldsymbol{\theta} \boldsymbol{\theta}, T}(\boldsymbol{\theta})$ in Lemma 3 below, yields $\dot{L}_{\boldsymbol{\theta} \boldsymbol{\theta}, T} \rightarrow \mathcal{J}\left(\boldsymbol{\theta}_{0}\right)$ a.s. as $T \rightarrow \infty$. This and the invertibility of $\mathcal{J}\left(\boldsymbol{\theta}_{0}\right)$ obtained from Assumption 2 and the result $\mathcal{J}\left(\boldsymbol{\theta}_{0}\right)=-\mathcal{I}\left(\boldsymbol{\theta}_{0}\right)$ established below in Lemma 2 implies that, for all $T$ sufficiently large, $\dot{L}_{\boldsymbol{\theta} \boldsymbol{\theta}, T}$ is also invertible (a.s.) and $\dot{L}_{\boldsymbol{\theta} \theta, T}^{-1} \rightarrow \mathcal{J}\left(\boldsymbol{\theta}_{0}\right)^{-1}$ a.s. as $T \rightarrow \infty$. Multiplying the mean value expansion (16) with the Moore-Penrose inverse $\dot{L}_{\boldsymbol{\theta} \boldsymbol{\theta}, T}^{+}$of $\dot{L}_{\boldsymbol{\theta} \boldsymbol{\theta}, T}$ (this inverse exists for all $T$ ) and rearranging we obtain

$$
\begin{aligned}
T^{1 / 2}\left(\hat{\boldsymbol{\theta}}_{T}-\boldsymbol{\theta}_{0}\right)= & \left(I_{\operatorname{dim} \boldsymbol{\theta}}-\dot{L}_{\boldsymbol{\theta} \boldsymbol{\theta}, T}^{+} \dot{L}_{\boldsymbol{\theta} \boldsymbol{\theta}, T}\right) T^{1 / 2}\left(\hat{\boldsymbol{\theta}}_{T}-\boldsymbol{\theta}_{0}\right)+\dot{L}_{\boldsymbol{\theta} \boldsymbol{\theta}, T}^{+} T^{1 / 2} L_{\boldsymbol{\theta}, T}\left(\hat{\boldsymbol{\theta}}_{T}\right) \\
& -\dot{L}_{\boldsymbol{\theta} \boldsymbol{\theta}, T}^{+} T^{1 / 2} L_{\boldsymbol{\theta}, T}\left(\boldsymbol{\theta}_{0}\right) .
\end{aligned}
$$


The first two terms on the right hand side of (17) converge to zero a.s. (for the first term, this follows from the fact that for all $T$ sufficiently large $\dot{L}_{\boldsymbol{\theta} \boldsymbol{\theta}, T}$ is invertible; for the second one, this holds because $\hat{\boldsymbol{\theta}}_{T}$ being a maximizer of $L_{T}(\theta)$ and $\boldsymbol{\theta}_{0}$ being an interior point of $\Theta_{0}$ yield $L_{\boldsymbol{\theta}, T}\left(\hat{\boldsymbol{\theta}}_{T}\right)=0$ for all $T$ sufficiently large). Furthermore, the eventual a.s. invertibility of $\dot{L}_{\boldsymbol{\theta} \boldsymbol{\theta}, T}$ also means that $\dot{L}_{\boldsymbol{\theta} \boldsymbol{\theta}, T}^{+}-\mathcal{J}\left(\boldsymbol{\theta}_{0}\right)^{-1} \rightarrow 0$ a.s. Hence, (17) becomes

$$
T^{1 / 2}\left(\hat{\boldsymbol{\theta}}_{T}-\boldsymbol{\theta}_{0}\right)=o_{1}(1)-\left(\mathcal{J}\left(\boldsymbol{\theta}_{0}\right)^{-1}+o_{2}(1)\right) T^{1 / 2} L_{\boldsymbol{\theta}, T}\left(\boldsymbol{\theta}_{0}\right),
$$

where $o_{1}(1)$ and $o_{2}(1)$ (a vector- and a matrix-valued process, respectively) converge to zero a.s. Combining this with the result of Lemma 1 below and the property $\mathcal{J}\left(\boldsymbol{\theta}_{0}\right)=$ $-\mathcal{I}\left(\boldsymbol{\theta}_{0}\right)$ (see Lemma 2 below) completes the proof.

Lemma 1. Under the assumptions of Theorem 3, $T^{1 / 2} \frac{\partial}{\partial \boldsymbol{\theta}} L_{T}\left(\boldsymbol{\theta}_{0}\right) \stackrel{d}{\rightarrow} N\left(0, \mathcal{I}\left(\boldsymbol{\theta}_{0}\right)\right)$, where $\mathcal{I}\left(\boldsymbol{\theta}_{0}\right)=E\left[\frac{\partial l_{t}\left(\boldsymbol{\theta}_{0}\right)}{\partial \boldsymbol{\theta}} \frac{\partial l_{t}\left(\boldsymbol{\theta}_{0}\right)}{\partial \boldsymbol{\theta}^{\prime}}\right]$ is finite.

Proof. We begin by deriving the score vectors (of a single observation) with respect to parameters $\boldsymbol{\vartheta}$ and $\boldsymbol{\alpha}$. To this end, first note that $l_{t}(\boldsymbol{\theta})$ can be expressed as

$$
l_{t}(\boldsymbol{\theta})=\log \left(\sum_{m=1}^{M} \alpha_{m} \mathrm{n}_{d(p+1)}\left(\left(y_{t}, \boldsymbol{y}_{t-1}\right) ; \boldsymbol{\vartheta}_{m}\right)\right)-\log \left(\sum_{m=1}^{M} \alpha_{m} \mathrm{n}_{d p}\left(\boldsymbol{y}_{t-1} ; \boldsymbol{\vartheta}_{m}\right)\right) .
$$

Next, introduce the notation

$$
\begin{aligned}
l_{\alpha_{m}, t}^{(1)}\left(y_{t}, \boldsymbol{y}_{t-1}\right) & =\frac{\mathrm{n}_{d(p+1)}\left(\left(y_{t}, \boldsymbol{y}_{t-1}\right) ; \boldsymbol{\vartheta}_{m}\right)-\mathrm{n}_{d(p+1)}\left(\left(y_{t}, \boldsymbol{y}_{t-1}\right) ; \boldsymbol{\vartheta}_{M}\right)}{\sum_{n=1}^{M} \alpha_{n} \mathrm{n}_{d(p+1)}\left(\left(y_{t}, \boldsymbol{y}_{t-1}\right) ; \boldsymbol{\vartheta}_{n}\right)} \\
l_{\alpha_{m}, t}^{(2)}\left(\boldsymbol{y}_{t-1}\right) & =\frac{\mathrm{n}_{d p}\left(\boldsymbol{y}_{t-1} ; \boldsymbol{\vartheta}_{m}\right)-\mathrm{n}_{d p}\left(\boldsymbol{y}_{t-1} ; \boldsymbol{\vartheta}_{M}\right)}{\sum_{n=1}^{M} \alpha_{n} \mathrm{n}_{d p}\left(\boldsymbol{y}_{t-1} ; \boldsymbol{\vartheta}_{n}\right)} \\
l_{\boldsymbol{\vartheta}_{m}, t}^{(1)}\left(y_{t}, \boldsymbol{y}_{t-1}\right) & =\frac{\alpha_{m}}{\sum_{n=1}^{M} \alpha_{n} \mathrm{n}_{d(p+1)}\left(\left(y_{t}, \boldsymbol{y}_{t-1}\right) ; \boldsymbol{\vartheta}_{n}\right)} \frac{\partial}{\partial \boldsymbol{\vartheta}_{m}} \mathrm{n}_{d(p+1)}\left(\left(y_{t}, \boldsymbol{y}_{t-1}\right) ; \boldsymbol{\vartheta}_{m}\right) \\
& =\frac{\alpha_{m} \mathrm{n}_{d(p+1)}\left(\left(y_{t}, \boldsymbol{y}_{t-1}\right) ; \boldsymbol{\vartheta}_{m}\right)}{\sum_{n=1}^{M} \alpha_{n} \mathrm{n}_{d(p+1)}\left(\left(y_{t}, \boldsymbol{y}_{t-1}\right) ; \boldsymbol{\vartheta}_{n}\right)} \frac{\partial}{\partial \boldsymbol{\vartheta}_{m}} \log \mathrm{n}_{d(p+1)}\left(\left(y_{t}, \boldsymbol{y}_{t-1}\right) ; \boldsymbol{\vartheta}_{m}\right) \\
l_{\boldsymbol{\vartheta}_{m}, t}^{(2)}\left(\boldsymbol{y}_{t-1}\right) & =\frac{\alpha_{m}}{\sum_{n=1}^{M} \alpha_{n} \mathrm{n}_{d p}\left(\boldsymbol{y}_{t-1} ; \boldsymbol{\vartheta}_{n}\right)} \frac{\partial}{\partial \boldsymbol{\vartheta}_{m}} \mathrm{n}_{d p}\left(\boldsymbol{y}_{t-1} ; \boldsymbol{\vartheta}_{m}\right) \\
& =\frac{\alpha_{m} \mathrm{n}_{d p}\left(\boldsymbol{y}_{t-1} ; \boldsymbol{\vartheta}_{m}\right)}{\sum_{n=1}^{M} \alpha_{n} \mathrm{n}_{d p}\left(\boldsymbol{y}_{t-1} ; \boldsymbol{\vartheta}_{n}\right)} \frac{\partial}{\partial \boldsymbol{\vartheta}_{m}} \log \mathrm{n}_{d p}\left(\boldsymbol{y}_{t-1} ; \boldsymbol{\vartheta}_{m}\right)
\end{aligned}
$$

where $m=1, \ldots, M-1$ in the first two quantities defined, and $m=1, \ldots, M$ in the last two. These quantities also depend on $\boldsymbol{\theta}$, but for brevity we have suppressed this dependence. For the corresponding quantities evaluated at $\boldsymbol{\theta}=\boldsymbol{\theta}_{0}$, we use the notation 
$l_{\alpha_{m}, t, 0}^{(1)}\left(y_{t}, \boldsymbol{y}_{t-1}\right), l_{\alpha_{m}, t, 0}^{(2)}\left(\boldsymbol{y}_{t-1}\right), l_{\boldsymbol{\vartheta}_{m}, t, 0}^{(1)}\left(y_{t}, \boldsymbol{y}_{t-1}\right)$, and $l_{\boldsymbol{\vartheta}_{m}, t, 0}^{(2)}\left(\boldsymbol{y}_{t-1}\right)$. Now, with straightforward differentiation of (18) we obtain the partial derivative with respect to $\alpha_{m}$ as

$$
\frac{\partial}{\partial \alpha_{m}} l_{t}(\boldsymbol{\theta})=l_{\alpha_{m}, t}^{(1)}\left(y_{t}, \boldsymbol{y}_{t-1}\right)-l_{\alpha_{m}, t}^{(2)}\left(\boldsymbol{y}_{t-1}\right), \quad m=1, \ldots, M-1,
$$

and with respect to $\boldsymbol{\vartheta}_{m}$ as

$$
\frac{\partial}{\partial \boldsymbol{\vartheta}_{m}} l_{t}(\boldsymbol{\theta})=l_{\boldsymbol{\vartheta}_{m}, t}^{(1)}\left(y_{t}, \boldsymbol{y}_{t-1}\right)-l_{\boldsymbol{\vartheta}_{m}, t}^{(2)}\left(\boldsymbol{y}_{t-1}\right), \quad m=1, \ldots, M
$$

Making use of the identities

$$
\sum_{m=1}^{M} \alpha_{m} \mathrm{n}_{d(p+1)}\left(\left(y_{t}, \boldsymbol{y}_{t-1}\right) ; \boldsymbol{\vartheta}_{m}\right)=\sum_{m=1}^{M} \alpha_{m, t} \mathrm{n}_{d}\left(y_{t} \mid \boldsymbol{y}_{t-1} ; \boldsymbol{\vartheta}_{m}\right) \sum_{m=1}^{M} \alpha_{m} \mathrm{n}_{d p}\left(\boldsymbol{y}_{t-1} ; \boldsymbol{\vartheta}_{m}\right)
$$

and

$$
\mathrm{n}_{d(p+1)}\left(\left(y_{t}, \boldsymbol{y}_{t-1}\right) ; \boldsymbol{\vartheta}_{m}\right)=\mathrm{n}_{d}\left(y_{t} \mid \boldsymbol{y}_{t-1} ; \boldsymbol{\vartheta}_{m}\right) \mathrm{n}_{d p}\left(\boldsymbol{y}_{t-1} ; \boldsymbol{\vartheta}_{m}\right)
$$

together with the definition of $\alpha_{m, t}$ these can alternatively be written as

$$
\begin{gathered}
\frac{\partial}{\partial \alpha_{m}} l_{t}(\boldsymbol{\theta})=\frac{1}{\sum_{n=1}^{M} \alpha_{n, t} \mathrm{n}_{d}\left(y_{t} \mid \boldsymbol{y}_{t-1} ; \boldsymbol{\vartheta}_{n}\right)}\left[\frac{\alpha_{m, t}}{\alpha_{m}} \mathrm{n}_{d}\left(y_{t} \mid \boldsymbol{y}_{t-1} ; \boldsymbol{\vartheta}_{m}\right)-\frac{\alpha_{M, t}}{\alpha_{M}} \mathrm{n}_{d}\left(y_{t} \mid \boldsymbol{y}_{t-1} ; \boldsymbol{\vartheta}_{M}\right)\right] \\
-\frac{\alpha_{m, t}}{\alpha_{m}}+\frac{\alpha_{M, t}}{\alpha_{M}}, \quad m=1, \ldots, M-1,
\end{gathered}
$$

and

$$
\begin{array}{r}
\frac{\partial}{\partial \boldsymbol{\vartheta}_{m}} l_{t}(\boldsymbol{\theta})=\frac{\alpha_{m, t} \mathrm{n}_{d}\left(y_{t} \mid \boldsymbol{y}_{t-1} ; \boldsymbol{\vartheta}_{m}\right)}{\sum_{n=1}^{M} \alpha_{n, t} \mathrm{n}_{d}\left(y_{t} \mid \boldsymbol{y}_{t-1} ; \boldsymbol{\vartheta}_{n}\right)} \frac{\partial}{\partial \boldsymbol{\vartheta}_{m}} \log \mathrm{n}_{d(p+1)}\left(\left(y_{t}, \boldsymbol{y}_{t-1}\right) ; \boldsymbol{\vartheta}_{m}\right) \\
-\alpha_{m, t} \frac{\partial}{\partial \boldsymbol{\vartheta}_{m}} \log \mathrm{n}_{d p}\left(\boldsymbol{y}_{t-1} ; \boldsymbol{\vartheta}_{m}\right), \quad m=1, \ldots, M
\end{array}
$$

As the process $y_{t}$ is assumed to be stationary and ergodic, so is also the score vector. We next establish that $\partial l_{t}\left(\boldsymbol{\theta}_{0}\right) / \partial \boldsymbol{\theta}$ is square integrable. To this end, conclude from (19) that $\left|\partial l_{t}\left(\boldsymbol{\theta}_{0}\right) / \partial \alpha_{m}\right| \leq c<\infty$, so that it suffices to consider $\partial l_{t}\left(\boldsymbol{\theta}_{0}\right) / \partial \boldsymbol{\vartheta}$. Thus, the desired result is obtained by showing that $l_{\boldsymbol{\vartheta}_{m}, t, 0}^{(1)}\left(y_{t}, \boldsymbol{y}_{t-1}\right)$ and $l_{\boldsymbol{\vartheta}_{m}, t, 0}^{(2)}\left(\boldsymbol{y}_{t-1}\right)$ are square integrable. To establish this, note that

$$
\begin{aligned}
\left|l_{\boldsymbol{\vartheta}_{m}, t, 0}^{(1)}\left(y_{t}, \boldsymbol{y}_{t-1}\right)\right|^{2} & \leq \frac{\alpha_{m, 0} \mathrm{n}_{d(p+1)}\left(\left(y_{t}, \boldsymbol{y}_{t-1}\right) ; \boldsymbol{\vartheta}_{m, 0}\right)}{\sum_{n=1}^{M} \alpha_{n, 0} \mathrm{n}_{d(p+1)}\left(\left(y_{t}, \boldsymbol{y}_{t-1}\right) ; \boldsymbol{\vartheta}_{n, 0}\right)}\left|\frac{\partial}{\partial \boldsymbol{\vartheta}_{m}} \log \mathrm{n}_{d(p+1)}\left(\left(y_{t}, \boldsymbol{y}_{t-1}\right) ; \boldsymbol{\vartheta}_{m, 0}\right)\right|^{2}, \\
\left|l_{\boldsymbol{\vartheta}_{m}, t, 0}^{(2)}\left(\boldsymbol{y}_{t-1}\right)\right|^{2} & \leq \frac{\alpha_{m, 0} \mathrm{n}_{d p}\left(\boldsymbol{y}_{t-1} ; \boldsymbol{\vartheta}_{m, 0}\right)}{\sum_{n=1}^{M} \alpha_{n, 0} \mathrm{n}_{d p}\left(\boldsymbol{y}_{t-1} ; \boldsymbol{\vartheta}_{n, 0}\right)}\left|\frac{\partial}{\partial \boldsymbol{\vartheta}_{m}} \log \mathrm{n}_{d p}\left(\boldsymbol{y}_{t-1} ; \boldsymbol{\vartheta}_{m, 0}\right)\right|^{2} .
\end{aligned}
$$

Hence,

$$
E\left[\left|l_{\boldsymbol{\vartheta}_{m}, t, 0}^{(1)}\left(y_{t}, \boldsymbol{y}_{t-1}\right)\right|^{2}\right] \leq \alpha_{m, 0} \int\left|\frac{\partial}{\partial \boldsymbol{\vartheta}_{m}} \log \mathrm{n}_{d(p+1)}\left((y, \boldsymbol{y}) ; \boldsymbol{\vartheta}_{m, 0}\right)\right|^{2} \mathrm{n}_{d(p+1)}\left((y, \boldsymbol{y}) ; \boldsymbol{\vartheta}_{m, 0}\right) d y d \boldsymbol{y}
$$


which is finite because the integral is the expectation of the squared norm of the score of $\boldsymbol{\vartheta}_{m}$ corresponding to the density $\mathrm{n}_{d(p+1)}\left((y, \boldsymbol{y}) ; \boldsymbol{\vartheta}_{m, 0}\right)$. In a similar manner it is seen that $E\left[\left|l_{\boldsymbol{\vartheta}_{m}, t, 0}^{(2)}\left(\boldsymbol{y}_{t-1}\right)\right|^{2}\right]<\infty$. Thus, we have shown that that $\partial l_{t}\left(\boldsymbol{\theta}_{0}\right) / \partial \boldsymbol{\theta}$ is square integrable.

For the martingale difference property, let $\alpha_{n, t, 0}$ signify $\alpha_{n, t}$ evaluated at $\boldsymbol{\theta}=\boldsymbol{\theta}_{0}$, and notice that $E\left[\cdot \mid \mathcal{F}_{t-1}\right]=\int \cdot \sum_{n=1}^{M} \alpha_{n, t, 0} \mathbf{n}_{d}\left(y \mid \boldsymbol{y} ; \boldsymbol{\vartheta}_{n, 0}\right) d y$. Concerning the score with respect to $\boldsymbol{\alpha}$, taking conditional expectations it is immediately seen from (19) that $E\left[\partial l_{t}\left(\boldsymbol{\theta}_{0}\right) / \partial \alpha_{m} \mid \mathcal{F}_{t-1}\right]=0$ holds. As for the score with respect to $\boldsymbol{\vartheta}_{m}$, use (24) and the fact $\log \mathrm{n}_{d(p+1)}\left(\left(y_{t}, \boldsymbol{y}_{t-1}\right) ; \boldsymbol{\vartheta}_{m}\right)=\log \mathrm{n}_{d}\left(y_{t} \mid \boldsymbol{y}_{t-1} ; \boldsymbol{\vartheta}_{m}\right)+\log \mathrm{n}_{d p}\left(\boldsymbol{y}_{t-1} ; \boldsymbol{\vartheta}_{m}\right)$ to obtain

$$
\begin{aligned}
& \frac{\partial}{\partial \boldsymbol{\vartheta}_{m}} l_{t}(\boldsymbol{\theta}) \sum_{n=1}^{M} \alpha_{n, t, 0} \mathrm{n}_{d}\left(y_{t} \mid \boldsymbol{y}_{t-1} ; \boldsymbol{\vartheta}_{n, 0}\right) \\
= & \alpha_{m, t, 0} \mathrm{n}_{d}\left(y_{t} \mid \boldsymbol{y}_{t-1} ; \boldsymbol{\vartheta}_{m, 0}\right) \frac{\partial}{\partial \boldsymbol{\vartheta}_{m}} \log \mathrm{n}_{d}\left(y_{t} \mid \boldsymbol{y}_{t-1} ; \boldsymbol{\vartheta}_{m, 0}\right) \\
& \left.+\alpha_{m, t, 0} \frac{\partial}{\partial \boldsymbol{\vartheta}_{m}} \log \mathrm{n}_{d p}\left(\boldsymbol{y}_{t-1}\right) ; \boldsymbol{\vartheta}_{m, 0}\right)\left[\mathrm{n}_{d}\left(y_{t} \mid \boldsymbol{y}_{t-1} ; \boldsymbol{\vartheta}_{m, 0}\right)-\sum_{n=1}^{M} \alpha_{n, t, 0} \mathrm{n}_{d}\left(y_{t} \mid \boldsymbol{y}_{t-1} ; \boldsymbol{\vartheta}_{n, 0}\right)\right] .
\end{aligned}
$$

Integrating over $y_{t}$ results in a zero vector because $\frac{\partial}{\partial \boldsymbol{\vartheta}_{m}} \log \mathrm{n}_{d}\left(y_{t} \mid \boldsymbol{y}_{t-1} ; \boldsymbol{\vartheta}_{m, 0}\right)$ is the score vector corresponding to the density $\mathrm{n}_{d}\left(y_{t} \mid \boldsymbol{y}_{t-1} ; \boldsymbol{\vartheta}_{m, 0}\right)$, so that also $E\left[\partial l_{t}\left(\boldsymbol{\theta}_{0}\right) / \partial \boldsymbol{\vartheta}_{m} \mid\right.$ $\left.\mathcal{F}_{t-1}\right]=0$ holds.

The stated asymptotic normality now follows from the central limit theorem for stationary and ergodic martingale differences (see Billingsley (1961)).

Lemma 2. Under the assumptions of Theorem 3, $\mathcal{J}\left(\boldsymbol{\theta}_{0}\right)=-\mathcal{I}\left(\boldsymbol{\theta}_{0}\right)$.

Proof. With straightforward differentiation, the required second partial derivatives can be expressed as

$$
\begin{aligned}
\frac{\partial^{2}}{\partial \alpha_{m} \partial \alpha_{n}} l_{t}(\boldsymbol{\theta})= & -l_{\alpha_{m}, t}^{(1)}\left(y_{t}, \boldsymbol{y}_{t-1}\right) l_{\alpha_{n}, t}^{(1)}\left(y_{t}, \boldsymbol{y}_{t-1}\right)+l_{\alpha_{m}, t}^{(2)}\left(\boldsymbol{y}_{t-1}\right) l_{\alpha_{n}, t}^{(2)}\left(\boldsymbol{y}_{t-1}\right) \\
\frac{\partial^{2}}{\partial \boldsymbol{\vartheta}_{m} \partial \boldsymbol{\vartheta}_{n}^{\prime}} l_{t}(\boldsymbol{\theta})= & -l_{\boldsymbol{\vartheta}_{m}, t}^{(1)}\left(y_{t}, \boldsymbol{y}_{t-1}\right) l_{\boldsymbol{\vartheta}_{n}, t}^{(1)}\left(y_{t}, \boldsymbol{y}_{t-1}\right)^{\prime}+l_{\boldsymbol{\vartheta}_{m}, t}^{(2)}\left(\boldsymbol{y}_{t-1}\right) l_{\boldsymbol{\vartheta}_{n}, t}^{(2)}\left(\boldsymbol{y}_{t-1}\right)^{\prime} \\
& +\frac{\alpha_{m}}{\sum_{k=1}^{M} \alpha_{k} \mathrm{n}_{d(p+1)}\left(\left(y_{t}, \boldsymbol{y}_{t-1}\right) ; \boldsymbol{\vartheta}_{k}\right)} \frac{\partial^{2}}{\partial \boldsymbol{\vartheta}_{m} \partial \boldsymbol{\vartheta}_{n}^{\prime}} \mathrm{n}_{d(p+1)}\left(\left(y_{t}, \boldsymbol{y}_{t-1}\right) ; \boldsymbol{\vartheta}_{m}\right) \\
& -\frac{\alpha_{m}}{\sum_{k=1}^{M} \alpha_{k} \mathrm{n}_{d p}\left(\boldsymbol{y}_{t-1} ; \boldsymbol{\vartheta}_{k}\right)} \frac{\partial^{2}}{\partial \boldsymbol{\vartheta}_{m} \partial \boldsymbol{\vartheta}_{n}^{\prime}} \mathrm{n}_{d p}\left(\boldsymbol{y}_{t-1} ; \boldsymbol{\vartheta}_{m}\right) \\
\frac{\partial^{2}}{\partial \boldsymbol{\vartheta}_{m} \partial \alpha_{n}} l_{t}(\boldsymbol{\theta})= & -l_{\alpha_{n}, t}^{(1)}\left(y_{t}, \boldsymbol{y}_{t-1}\right) l_{\boldsymbol{\vartheta}_{m}, t}^{(1)}\left(y_{t}, \boldsymbol{y}_{t-1}\right)+l_{\alpha_{n}, t}^{(2)}\left(\boldsymbol{y}_{t-1}\right) l_{\boldsymbol{\vartheta}_{m}, t}^{(2)}\left(\boldsymbol{y}_{t-1}\right), \\
\frac{\partial^{2}}{\partial \boldsymbol{\vartheta}_{m} \partial \alpha_{m}} l_{t}(\boldsymbol{\theta})= & -l_{\alpha_{m}, t}^{(1)}\left(y_{t}, \boldsymbol{y}_{t-1}\right) l_{\boldsymbol{\vartheta}_{m}, t}^{(1)}\left(y_{t}, \boldsymbol{y}_{t-1}\right)+l_{\alpha_{m}, t}^{(2)}\left(\boldsymbol{y}_{t-1}\right) l_{\boldsymbol{\vartheta}_{m}, t}^{(2)}\left(\boldsymbol{y}_{t-1}\right) \\
& +\alpha_{m}^{-1} l_{\boldsymbol{\vartheta}_{m}, t}^{(1)}\left(y_{t}, \boldsymbol{y}_{t-1}\right)-\alpha_{m}^{-1} l_{\boldsymbol{\vartheta}_{m}, t}^{(2)}\left(\boldsymbol{y}_{t-1}\right),
\end{aligned}
$$


where in the first expression $m, n=1, \ldots, M-1$; in the second $m, n=1, \ldots, M$; in the third $m=1, \ldots, M, n=1, \ldots, M-1$, and $m \neq n$; and in the fourth $m=1, \ldots, M-1$. Using these expressions together with those for the first partial derivatives of $l_{t}(\boldsymbol{\theta})$ given in (19) and (20), the result $\mathcal{J}\left(\boldsymbol{\theta}_{0}\right)=E\left[\partial^{2} l_{t}\left(\boldsymbol{\theta}_{0}\right) / \partial \boldsymbol{\theta} \partial \boldsymbol{\theta}^{\prime}\right]=-E\left[\left(\partial l_{t}\left(\boldsymbol{\theta}_{0}\right) / \partial \boldsymbol{\theta}\right)\left(\partial l_{t}\left(\boldsymbol{\theta}_{0}\right) / \partial \boldsymbol{\theta}^{\prime}\right)\right]=$ $-\mathcal{I}\left(\boldsymbol{\theta}_{0}\right)$ can be established using elementary but tedious calculations. For brevity, we omit the details, which are available in the Supplementary Appendix.

Lemma 3. Under the assumptions of Theorem 3, $\sup _{\boldsymbol{\theta} \in \Theta_{0}}\left|L_{\boldsymbol{\theta} \boldsymbol{\theta}, T}(\boldsymbol{\theta})-\mathcal{J}(\boldsymbol{\theta})\right| \rightarrow 0$ a.s., where $\mathcal{J}(\boldsymbol{\theta})=E\left[l_{\boldsymbol{\theta}, t}(\boldsymbol{\theta})\right]$ is continuous at $\boldsymbol{\theta}_{0}$.

Proof. As the process $y_{t}$ is assumed to be stationary and ergodic, from the expressions of the components of $l_{\boldsymbol{\theta} \boldsymbol{\theta}, t}(\boldsymbol{\theta})$ given at the beginning of the proof of Lemma 2 (see also the equations after (18)) it follows that $l_{\boldsymbol{\theta} \boldsymbol{\theta}, t}(\boldsymbol{\theta})$ forms a stationary ergodic sequence of random variables that are continuous in $\boldsymbol{\theta}$ over $\Theta_{0}$. The desired result thus follows from Ranga Rao (1962) if we establish that $E\left[\sup _{\boldsymbol{\theta} \in \Theta_{0}}\left|l_{\boldsymbol{\theta} \boldsymbol{\theta}, t}(\boldsymbol{\theta})\right|\right]$ is finite. To this end, first note that

$$
\alpha_{m, t}=\frac{\alpha_{m} \mathrm{n}_{d p}\left(\boldsymbol{y}_{t-1} ; \boldsymbol{\vartheta}_{m}\right)}{\sum_{n=1}^{M} \alpha_{n} \mathrm{n}_{d p}\left(\boldsymbol{y}_{t-1} ; \boldsymbol{\vartheta}_{n}\right)}<1 \quad \text { and } \quad \frac{\alpha_{m} \mathrm{n}_{d(p+1)}\left(\left(y_{t}, \boldsymbol{y}_{t-1}\right) ; \boldsymbol{\vartheta}_{m}\right)}{\sum_{n=1}^{M} \alpha_{n} \mathrm{n}_{d(p+1)}\left(\left(y_{t}, \boldsymbol{y}_{t-1}\right) ; \boldsymbol{\vartheta}_{n}\right)}<1
$$

for $m=1, \ldots, M$, and observe that the set $\Theta_{0}$ can be chosen small enough to ensure that $\alpha_{m}, m=1, \ldots, M$, are bounded away from zero on $\Theta_{0}$. Using these facts and the definitions of $l_{\alpha_{m}, t}^{(1)}\left(y_{t}, \boldsymbol{y}_{t-1}\right), l_{\alpha_{m}, t}^{(2)}\left(\boldsymbol{y}_{t-1}\right), l_{\boldsymbol{\vartheta}_{m}, t}^{(1)}\left(y_{t}, \boldsymbol{y}_{t-1}\right)$, and $l_{\boldsymbol{\vartheta}_{m}, t}^{(2)}\left(\boldsymbol{y}_{t-1}\right)$ (see the equations after (18)) it can then be seen that

$$
\begin{aligned}
&\left|l_{\alpha_{m}, t}^{(1)}\left(y_{t}, \boldsymbol{y}_{t-1}\right)\right| \leq C \text { and }\left|l_{\alpha_{m}, t}^{(2)}\left(\boldsymbol{y}_{t-1}\right)\right| \leq C \quad(m=1, \ldots, M-1), \\
&\left|l_{\boldsymbol{\vartheta}_{m}, t}^{(1)}\left(y_{t}, \boldsymbol{y}_{t-1}\right)\right| \leq\left|\frac{\partial}{\partial \boldsymbol{\vartheta}_{m}} \log \mathrm{n}_{d(p+1)}\left(\left(y_{t}, \boldsymbol{y}_{t-1}\right) ; \boldsymbol{\vartheta}_{m}\right)\right| \quad(m=1, \ldots, M),(m=1, \ldots, M), \\
&\left|l_{\boldsymbol{\vartheta}_{m}, t}^{(2)}\left(\boldsymbol{y}_{t-1}\right)\right| \leq\left|\frac{\partial}{\partial \boldsymbol{\vartheta}_{m}} \log \mathrm{n}_{d p}\left(\boldsymbol{y}_{t-1} ; \boldsymbol{\vartheta}_{m}\right)\right| \quad(m=1, \quad
\end{aligned}
$$

for some $C<\infty$ and all $\boldsymbol{\theta} \in \Theta_{0}$. These upper bounds, the expressions of the second partial derivatives of $l_{t}(\boldsymbol{\theta})$ at the beginning of the proof of Lemma 2, the relation

$$
\begin{aligned}
\frac{\partial^{2}}{\partial \boldsymbol{\vartheta}_{m} \partial \boldsymbol{\vartheta}_{m}^{\prime}} \mathrm{n}_{d p}\left(\boldsymbol{y}_{t-1} ; \boldsymbol{\vartheta}_{m}\right)= & \mathrm{n}_{d p}\left(\boldsymbol{y}_{t-1} ; \boldsymbol{\vartheta}_{m}\right) \frac{\partial}{\partial \boldsymbol{\vartheta}_{m}} \log \mathrm{n}_{d p}\left(\boldsymbol{y}_{t-1} ; \boldsymbol{\vartheta}_{m}\right) \frac{\partial}{\partial \boldsymbol{\vartheta}_{m}^{\prime}} \log \mathrm{n}_{d p}\left(\boldsymbol{y}_{t-1} ; \boldsymbol{\vartheta}_{m}\right) \\
& +\mathrm{n}_{d p}\left(\boldsymbol{y}_{t-1} ; \boldsymbol{\vartheta}_{m}\right) \frac{\partial^{2}}{\partial \boldsymbol{\vartheta}_{m} \partial \boldsymbol{\vartheta}_{m}^{\prime}} \log \mathrm{n}_{d p}\left(\boldsymbol{y}_{t-1} ; \boldsymbol{\vartheta}_{m}\right)
\end{aligned}
$$

and an analogous relation for the density $\mathrm{n}_{d(p+1)}\left(\left(y_{t}, \boldsymbol{y}_{t-1}\right) ; \boldsymbol{\vartheta}_{m}\right)$, can now be used to show that $E\left[\sup _{\boldsymbol{\theta} \in \Theta_{0}}\left|l_{\boldsymbol{\theta} \boldsymbol{\theta}, t}(\boldsymbol{\theta})\right|\right]<\infty$ holds as long as

$E\left[\sup _{\boldsymbol{\theta} \in \Theta_{0}}\left|\frac{\partial}{\partial \boldsymbol{\vartheta}_{m}} \log \mathrm{n}_{d p}\left(\boldsymbol{y}_{t-1} ; \boldsymbol{\vartheta}_{m}\right)\right|^{2}\right]<\infty$ and $E\left[\sup _{\boldsymbol{\theta} \in \Theta_{0}}\left|\frac{\partial^{2}}{\partial \boldsymbol{\vartheta}_{m} \partial \boldsymbol{\vartheta}_{m}^{\prime}} \log \mathrm{n}_{d p}\left(\boldsymbol{y}_{t-1} ; \boldsymbol{\vartheta}_{m}\right)\right|\right]<\infty$ 
together with analogous results for the density $\mathrm{n}_{d(p+1)}\left(\left(y_{t}, \boldsymbol{y}_{t-1}\right) ; \boldsymbol{\vartheta}_{m}\right)$, hold for $m=$ $1, \ldots, M$.

To establish the finiteness of these moments, first consider the partial derivatives of $\log \mathrm{n}_{d p}\left(\boldsymbol{y}_{t-1} ; \boldsymbol{\vartheta}_{m}\right)$, and for clarity let $\boldsymbol{\mu}\left(\boldsymbol{\vartheta}_{m}\right)\left(=\mathbf{1}_{p} \otimes \mu_{m}\right)$ and $\boldsymbol{\Sigma}\left(\boldsymbol{\vartheta}_{m}\right) \quad\left(=\boldsymbol{\Sigma}_{m, p}\right)$ denote the mean vector and covariance matrix of the $\mathrm{n}_{d p}\left(\boldsymbol{y}_{t-1} ; \boldsymbol{\vartheta}_{m}\right)$ distribution as functions of the parameter vector $\boldsymbol{\vartheta}_{m}$. From the equality $\mu_{m}=A_{m}^{-1}(1) \phi_{m, 0}$ and the expression of $\boldsymbol{\Sigma}\left(\boldsymbol{\vartheta}_{m}\right)$ in Lütkepohl (2005, eqn. (2.1.39)) as a function of $A_{m, i}, i=1, \ldots, p$, and $\boldsymbol{\Omega}_{m}$, and hence of $\boldsymbol{\vartheta}_{m}$, it follows that $\boldsymbol{\mu}\left(\boldsymbol{\vartheta}_{m}\right)$ and $\boldsymbol{\Sigma}\left(\boldsymbol{\vartheta}_{m}\right)$ are twice continuously differentiable functions of $\boldsymbol{\vartheta}_{m}$. Now, for each component $\vartheta_{m, i}$ of $\boldsymbol{\vartheta}_{m}\left(i=1, \ldots, \operatorname{dim} \boldsymbol{\vartheta}_{m}\right)$, straightforward differentiation gives

$$
\begin{aligned}
\frac{\partial}{\partial \vartheta_{m, i}} \log \mathbf{n}_{d p}\left(\boldsymbol{y}_{t-1} ; \boldsymbol{\vartheta}_{m}\right)=-\frac{1}{2} \operatorname{tr}\left(\boldsymbol{\Sigma}\left(\boldsymbol{\vartheta}_{m}\right)^{-1} \frac{\partial \boldsymbol{\Sigma}\left(\boldsymbol{\vartheta}_{m}\right)}{\partial \vartheta_{m, i}}\right)+\left(\boldsymbol{y}_{t-1}-\boldsymbol{\mu}\left(\boldsymbol{\vartheta}_{m}\right)\right)^{\prime} \boldsymbol{\Sigma}\left(\boldsymbol{\vartheta}_{m}\right)^{-1} \frac{\partial \boldsymbol{\mu}\left(\boldsymbol{\vartheta}_{m}\right)}{\partial \vartheta_{m, i}} \\
+\frac{1}{2}\left[\operatorname{vec}\left(\left(\boldsymbol{y}_{t-1}-\boldsymbol{\mu}\left(\boldsymbol{\vartheta}_{m}\right)\right)\left(\boldsymbol{y}_{t-1}-\boldsymbol{\mu}\left(\boldsymbol{\vartheta}_{m}\right)\right)^{\prime}\right)\right]^{\prime} \operatorname{vec}\left(\boldsymbol{\Sigma}\left(\boldsymbol{\vartheta}_{m}\right)^{-1} \frac{\partial \boldsymbol{\Sigma}\left(\boldsymbol{\vartheta}_{m}\right)}{\partial \vartheta_{m, i}} \boldsymbol{\Sigma}\left(\boldsymbol{\vartheta}_{m}\right)^{-1}\right) .
\end{aligned}
$$

As the set $\Theta_{0}$ can be assumed small enough to ensure that the functions of $\boldsymbol{\mu}\left(\boldsymbol{\vartheta}_{m}\right)$ and $\boldsymbol{\Sigma}\left(\boldsymbol{\vartheta}_{m}\right)$ as well as their partial derivatives appearing in the last expression above are bounded on $\Theta_{0}, E\left[\sup _{\boldsymbol{\theta} \in \Theta_{0}}\left|\frac{\partial}{\partial \boldsymbol{\vartheta}_{m}} \log \mathrm{n}_{d p}\left(\boldsymbol{y}_{t-1} ; \boldsymbol{\vartheta}_{m}\right)\right|^{2}\right]<\infty$ follows because $\boldsymbol{y}_{t}$ has finite fourth moments due to Theorem 1. The finiteness of the three other moment conditions follows in a similar fashion (details omitted) because $\boldsymbol{\mu}\left(\boldsymbol{\vartheta}_{m}\right)$ and $\boldsymbol{\Sigma}\left(\boldsymbol{\vartheta}_{m}\right)$ are twice continuously differentiable and $\boldsymbol{y}_{t}$ has finite moments of all orders due to Theorem 1. 


\section{References}

Anderson, T. W. (2003): An Introduction to Multivariate Statistical Analysis, 3rd edn. Wiley, Hoboken NJ.

Ang, A. And G. BeKAert (2002): "Regime switches in interest rates," Journal of Business and Economic Statistics, 20, 163-182.

Bec, F., A. Rahbek, and N. Shephard (2008): "The ACR model: a multivariate dynamic mixture autoregression," Oxford Bulletin of Economics and Statistics, 70, $583-618$.

Billingsley, P. (1961): "The Lindeberg-Levy theorem for martingales," Proceedings of the American Mathematical Society, 12, 788-792.

Carrasco, M., L. Hu, and W. Ploberger (2014): "Optimal tests for Markov switching parameters," Econometrica, 82, 765-784.

Cho, J. S. And H. White (2007): "Testing for regime switching," Econometrica, 75, $1671-1720$.

Christoffersen, P. F. (1998): "Evaluating interval forecasts," International Economic Review, 39, 841-862.

Dueker, M. J., Z. Psaradakis, M. Sola and F. Spagnolo (2011): "Multivariate contemporaneous-threshold autoregressive models," Journal of Econometrics, 160, $311-325$.

Dueker, M. J., M. Sola and F. Spagnolo (2007): "Contemporaneous threshold autoregressive models: estimation, testing and forecasting," Journal of Econometrics, $141,517-547$.

Fong, P. W., W. K. Li, C. W. Yau, and C. S. Wong (2007): "On a mixture vector autoregressive model," Canadian Journal of Statistics, 35, 135-150.

Glasbey, C. A. (2001): "Non-linear autoregressive time series with multivariate Gaussian mixtures as marginal distributions," Journal of the Royal Statistical Society: Series $C, 50,143-154$.

Kalliovirta, L. (2012): "Misspecification tests based on quantile residuals," Econometrics Journal, 15, 358-393. 
Kalliovirta, L., M. Meitz, and P. Saikkonen (2014): "Modeling the Euro-USD exchange rate with the Gaussian mixture autoregressive model," in J. Knif and B. Pape (eds.), Contributions to Mathematics, Statistics, Econometrics, and Finance: A Festschrift in Honour of Professor Seppo Pynnönen, Acta Wasaensia 296, University of Vaasa.

Kalliovirta, L., M. Meitz, and P. Saikkonen (2015): "A Gaussian mixture autoregressive model for univariate time series," Journal of Time Series Analysis, 36, $247-266$.

Kalliovirta, L., and P. Saikkonen (2010): "Reliable residuals for multivariate nonlinear time series models," unpublished revision of HECER Discussion Paper No. 247.

Lanne, M. (2006): "Nonlinear dynamics of interest rate and inflation," Journal of Applied Econometrics, 21, 1154-1168.

Krolzig, H.-M. (1997): Markov-Switching Vector Autoregressions Modelling, Statistical Inference and Applications to Business Cycle Analysis. Springer, Berlin.

Le, N. D., R. D. Martin, and A. E. Raftery (1996): "Modeling flat stretches, bursts, and outliers in time series using mixture transition distribution models," Journal of the American Statistical Association, 91, 1504-1515.

LÜtкepohl, H. (2005): New Introduction to Multiple Time Series Analysis. Springer, Berlin.

Meyn, S., And R. L. Tweedie (2009): Markov Chains and Stochastic Stability, 2nd edn. Cambridge University Press, Cambridge.

Pötscher, B. M., And I. R. Prucha (1991): "Basic structure of the asymptotic theory in dynamic nonlinear econometric models, Part I: Consistency and approximation concepts," Econometric Reviews, 10, 125-216.

RANGA RAO, R. (1962): "Relations between weak and uniform convergence of measures with applications," Annals of Mathematical Statistics, 33, 659-680.

Reinsel, G. C. (1997): Elements of Multivariate Time Series Analysis, 2nd edn. Springer, New York. 
Sims, C. A., D. F. Waggoner, And T. Zha (2008): "Methods for inference in large multiple equation Markov-switching models," Journal of Econometrics, 146, 255-274.

Teräsvirta, T., D. TJøstheim And C. W. J. Granger (2010): Modelling Nonlinear Economic Time Series. Oxford University Press, Oxford.

Tierney, L. (1994): "Markov chains for exploring posterior distributions," Annals of Statistics, 22, 1701-1762.

Tong, H. (2011): "Threshold models in time series analysis - 30 years on," Statistics and Its Interface, 4, 107-118.

Villani, M., R. Kohn, and P. Giordani (2009): "Regression density estimation using smooth adaptive Gaussian mixtures," Journal of Econometrics, 153, 155-173.

Wong, C. S., And W. K. Li (2000): "On a mixture autoregressive model," Journal of the Royal Statistical Society: Series B, 62, 95-115.

Wong, C. S., AND W. K. Li (2001a): "On a mixture autoregressive conditional heteroscedastic model," Journal of the American Statistical Association, 96, 982-995.

Wong, C. S., AND W. K. Li (2001b): "On a logistic mixture autoregressive model," Biometrika, 88, 833-846.

Yakowitz, S. J., And J. D. Spragins (1968): "On the identifiability of finite mixtures," Annals of Mathematical Statistics, 39, 209-214. 